\title{
Reinhardt cardinals and iterates of $V$
}

\author{
Farmer Schlutzenberg*
}

June 30, 2020

\begin{abstract}
Assume $\mathrm{ZF}(j)$ and there is a Reinhardt cardinal, as witnessed by the elementary embedding $j: V \rightarrow V$. We investigate the linear iterates $\left(N_{\alpha}, j_{\alpha}\right)$ of $(V, j)$, and their relationship to $(V, j)$, forcing and definability, including that for each infinite $\alpha$, every set is set-generic over $N_{\alpha}$, but $N_{\alpha}$ is not a set-ground.

Assume second order ZF. We prove that the existence of super Reinhardt cardinals and total Reinhardt cardinals is not affected by small forcing. And if $V[G]$ has a set of ordinals which is not in $V$, then $V[G]$ has no elementary embedding $j: V[G] \rightarrow M \subseteq V$ (even allowing $M$ to be illfounded). ${ }^{12}$
\end{abstract}

\section{Introduction}

A Reinhardt cardinal, introduced by William Reinhardt in [11] and [12], is the critical point of an elementary embedding $j: V \rightarrow V$. Kunen showed in [9] that if $V \models$ ZFC then there is no such $j$. So Reinhardt embeddings (that is, $j: V \rightarrow V$ ) are considered with background theory $\mathrm{ZF}(j)$ (for the basic definitions $\S 1.1$ below), possibly augmented with fragments of AC. We work throughout in $\mathrm{ZF}$ or variants thereof such as $\mathrm{ZF}(j)$ or $\mathrm{ZF}_{2}$, indicating any choice assumptions where they are adopted.

This paper primarily investigates the iterates of $V$ under an elemenetary $j: V \rightarrow V$. Let $M_{0}=(V, j)$. In Definition 3.2 we define the $\alpha$ th iterate $M_{\alpha}=\left(N_{\alpha}, j_{\alpha}\right)$ of $M_{0}$ for each $\alpha \in \mathrm{OR}$. These are analogous to the iterates of $\left(L\left(V_{\lambda+1}\right), k\right)$ in the context of $I_{0}$, investigated by Woodin in [20], and Mohammad Golshani posed a question of the nature of the $M_{\alpha}$ on the discussion board Mathoverflow.

In [7], many variants of and facts related to Kunen's ZFC inconsistency result are discussed. This paper makes a contribution toward understanding

\footnotetext{
*schlutze@uni-muenster.de

${ }^{1}$ Keywords: Reinhardt cardinal, iterate, HOD, definability, forcing, Axiom of Choice

2 This paper originates from a set of rather informal notes [16], published on arxiv.org (v1 of arXiv:2002.01215). The current paper is $\mathrm{v} n$ of (the same) arXiv:2002.01215, where $n>1$. Some of the results from the original notes, regarding definability and constructibility of embeddings $j: V_{\delta} \rightarrow V_{\delta}$ and related facts, and those on extenders under ZF and definability of $V$-critical points from a proper class of weak Löwenheim Skolem cardinals, are to appear in the papers [6] and [14]. The original notes together with some errata can be seen at https://sites.google.com/site/schlutzenberg/home-1, but it is in general better to refer to the current paper and the two just cited. Of course, the original notes also remain available on arxiv.org, as mentioned above.
} 
those issues under ZF, a question posed in [7]. The analysis of the iterates $M_{\alpha}$ will show that some of the conclusions proven in [7] fail in a strong way under $\mathrm{ZF}(j)+j: V \rightarrow V$. On the other hand, we are able to establish a partial ZF generalization of Woodin's ZFC theorem [7, Theorem 5] excluding elementary $j: V[G] \rightarrow V$.

We prove the following facts. In Theorem 3.14, we show that every set is set-generic over $N_{\alpha}$. The key to this is using a slight variant of a forcing due to Schindler (the latter being related to the Bukowsky forcing, and also to Vopenka and the extender algebra). In particular, for the iteration map $i_{0 \alpha}: V \rightarrow N_{\alpha}$, $i_{0 \alpha}\left\lceil V_{\beta}\right.$ is set-generic over $N_{\alpha}$, and this has the consequence that there are interesting generic extensions of $V$, including that $\operatorname{cr}(j)$ is a virtual Berkeley cardinal (see Definition 3.15 and Corollary 3.16). In Theorem 3.19, we show that, however, for $\alpha \geq \omega, N_{\alpha}$ is not a set-ground of $V$ (note $N_{n}=V$ for $n<\omega)$. It is well known that every set is also set-generic over HOD. We show in Theorem 3.7 that HOD $\subseteq N_{\omega}$, and in fact $\operatorname{Hull}^{\left(V, M_{\omega}\right)}\left(N_{\omega}\right)=N_{\omega}$ (where the hull is computed in $V$, with the predicate $M_{\omega}$ (including $j_{\omega}$ ) from parameters in $N_{\omega}$ ), but there are $\alpha \in \mathrm{OR}$ with $\mathrm{HOD} \nsubseteq N_{\alpha}$.

By [7, Theorem 12], under ZFC $\mathrm{ZF}_{2}$, if $j: V \rightarrow M$ is elementary with $M$ transitive then $V=\operatorname{Hull}^{V}(\operatorname{rg}(j))$. This fails trivially in the case that $(V, j) \models$ ZFR (where $M=V$ ), since then $\operatorname{Hull}^{V}(\operatorname{rg}(j))=\operatorname{rg}(j)$. However, because $\operatorname{Hull}^{V}\left(N_{\omega}\right)=N_{\omega}$, we get a non-trivial failure, as we have the iteration map $i_{0 \omega}: V \rightarrow N_{\omega}$, and $N_{\omega} \neq V$. We will also prove a stronger fact in Theorem 3.21 , that for every set $X, V \neq \operatorname{Hull}^{\left(V, M_{\omega}\right)}\left(N_{\omega} \cup X\right)$. At the same time we will establish a strengthening of Suzuki's theorem [17] that $j$ is not definable from parameters (which was strengthened in other ways in [7], [6] and [14]): we show that $j\left\lceil\mathrm{OR}\right.$ is not definable from parameters and the predicate $\left(N_{\omega}, j_{\omega}\right){ }^{3}$

It follows in particular that if there is a Reinhardt, then for all sets $X$, $V \neq \operatorname{HOD}(X)$. And if $V$ is total Reinhardt or there is a Berkeley cardinal, a similar proof gives that $V \neq \operatorname{HOD}_{A}(X)$ for any class $A$ and set $X$. But in fact much more is now known; see Remark 3.22.

In $\S 4$ we prove some facts regarding small forcing and (very) large cardinals. Theorem 4.6 asserts that if $\mathbb{P} \in V$ and $G$ is $(V, \mathbb{P})$-generic and $(V[G], j) \models$ ZFR+ "P is wellorderable and card $(\mathbb{P})^{+}$-DC holds", then $(V, j \mid V) \models$ ZFR. Assume $\mathrm{ZF}_{2}$. By Theorem 4.8, if $G$ is set-generic over $V$ and $V[G]$ has a super Reinhardt cardinal, then so does $V$. A similar fact is proven for total Reinhardtness. Theorem 4.4 is a variant of the $\mathrm{ZF}+\mathrm{DC}_{\delta}$ ground definability result of Gitman-Johnstone [2], in which we assume some DC in the generic extension, but not (at least not explicitly) in the ground model (but we assume that the forcing in question is small).

Theorem 2.3 is a generalization of Woodin's ZFC 2 theorem [7, Theorem 4] that if $G$ is set-generic over $V$ then there is no elementary $j: V[G] \rightarrow V$. We prove assuming $\mathrm{ZF}_{2}$ that if $G$ is set-generic over $V$ and $V[G]$ has some set of ordinals not in $V$, then there is no elementary $j: V[G] \rightarrow M \subseteq V$, even allowing $M$ to be illfounded. This fact appears to be new even in the ZFC context; the results in [7] seem to rely on stationary set combinatorics which does not seem to be available in this generality.

\footnotetext{
${ }^{3}$ The fact that $j\lceil\mathrm{OR}$ is not definable over $V$ from parameters (but without any extra predicate) is already a consequence of [7, Theorem 35], by which no elementary $k:$ HOD $\rightarrow$ HOD is definable from parameters (without assuming AC). In Remark 3.20 we also sketch an alternate proof of the latter fact.
} 
There are many questions that are open, and some of these are mentioned throughout. We would like to thank Gabriel Goldberg and Toshimichi Usuba for their feedback regarding certain questions in the notes [16] (upon which the material here is based), and other helpful suggestions.

\subsection{Basic definitions}

OR denotes the class of ordinals and Lim the limit ordinals.

The language $\mathcal{L}_{A_{1}, \ldots, A_{n}}$ of set theory with predicates $A_{1}, \ldots, A_{n}$ is the first order language with binary predicate symbol $\in$ and predicate symbols $A_{1}, \ldots, A_{n}$. The theory $\operatorname{ZF}(\vec{A})$ is the theory in $\mathcal{L}_{\in, \vec{A}}$ with all ZF axioms, allowing all formulas of $\mathcal{L}_{\in, \vec{A}}$ in the Separation and Collection schemes (so each $A_{i}$ represents a class).

ZFR (ZF+ Reinhardt) is the theory ZF $(\widetilde{j})+\widetilde{\widetilde{j}}: V \rightarrow V$ is $\Sigma_{1}$-elementary". And $\operatorname{ZFR}(A)$ is the theory $\operatorname{ZF}(\widetilde{j}, A)+{ }^{*} \tilde{j}:(V, A) \rightarrow(V, A)$ is $\Sigma_{1}$-elementary". Essentially by $[8, \operatorname{Proposition} 5.1], \operatorname{ZFR}(A)$ proves $\widetilde{j}$ is fully elementary (as a theorem scheme).

Second order set theory is denoted $\mathrm{ZF}_{2}$ (see [1]). Models are of the form $(V, \in, P)$, where $(V, \in) \models$ ZF and $P$ is a collection of classes/subsets of $V$, satisfying the $\mathrm{ZF}_{2}$ axioms. Given a transitive $W$ and $P \subseteq \mathcal{P}(W)$, we write $(W, P) \models \mathrm{ZF}_{2}$ iff $(W, \in, P) \models \mathrm{ZF}_{2}$. When we "work in $\mathrm{ZF}_{2}$ ", we mean that we work in such a model $W$, and all talk of proper classes refers to elements of $P$.

Let $\delta \in \operatorname{Lim}$. The cofinality of $\delta$, regularity, singularity are defined as usual (in terms of cofinal functions between ordinals). We say $\delta$ (or $V_{\delta}$ ) is inaccessible iff there is no $(\gamma, f)$ such that $\gamma<\delta$ and $f: V_{\gamma} \rightarrow \delta$ is cofinal in $\delta$.

Work in $\mathrm{ZF}_{2}$. Then $\kappa \in \mathrm{OR}$ is Reinhardt iff there is a class $j$ such that $(V, j) \models \mathrm{ZFR}$ and $\kappa=\operatorname{cr}(j)$. Following [1], $\kappa \in \mathrm{OR}$ is super-Reinhardt iff for every $\lambda \in \mathrm{OR}$ there is a class $j$ such that $(V, j) \models \mathrm{ZFR}$ and $\operatorname{cr}(j)=\kappa$ and $j(\kappa) \geq \lambda$. And OR is total Reinhardt if for every class $A$ there is $\kappa \in \mathrm{OR}$ such that $\kappa$ is $(<\mathrm{OR}, V, A)$-reflecting, meaning that for every $\lambda \in \mathrm{OR}$ there is a class $j$ such that $(V, j, A) \models \operatorname{ZFR}(A)$ and $\operatorname{cr}(j)=\kappa$ and $j(\kappa) \geq \lambda$. And $\delta$ is Berkeley if for all transitive sets $M$ with $\delta \in M$, and all $\eta<\delta$, there is an elementary $j: M \rightarrow M$ with $\eta<\operatorname{cr}(j)<\delta$.

Let $j: V_{\delta} \rightarrow V_{\delta}$ or $j: V \rightarrow V$ be $\Sigma_{1}$-elementary, for a limit $\delta$ or $\delta=$ OR. We write $\kappa_{0}(j)=\operatorname{cr}(j)$ and $\kappa_{n+1}(j)=j\left(\kappa_{n}(j)\right)$ and $\kappa_{\omega}(j)=\sup _{n<\omega} \kappa_{n}$.

Let $C$ be a class. Then $\operatorname{OD}_{C}(X)$ denotes the class of all sets $y$ such that $y$ is definable from the predicate $C$ and parameters in $\operatorname{OR} \cup X$. And $\operatorname{HOD}_{C}(X)$ denotes the class of all $y$ such that the transitive closure of $\{y\}$ is $\subseteq \operatorname{OD}_{C}(X)$. And $\mathrm{OD}(X)=\mathrm{OD}_{\emptyset}(X)$ and $\operatorname{HOD}(X)=\operatorname{HOD}_{\emptyset}(X)$. And for a structure $M$ and $X \subseteq M, \operatorname{Hull}^{M}(X)$ denotes the collection of elements $x \in M$ such that $x$ is definable over $M$ from (finitely many) parameters in $X$.

\section{$2 \quad$ Generic $j: V[G] \rightarrow M \subseteq V$}

The following fact, due to Woodin, is proved in [7, Theorem 5]:

Fact 2.1 (Woodin). Assume ZFC. Let $G$ be set-generic over $V$. Then there is no elementary $j: V[G] \rightarrow V$. 
Of course in the case that $G \in V$, this reduces to Kunen's inconsistency. Various generalizations of this result can also be seen in [7]; one in particular is that, by [7, Corollary 34], under ZF, if $G$ is set-generic over $V$, then there is no elementary $j: V[G] \rightarrow V$ which is definable from parameters over $V[G]$.

In this section, we generalize Fact 2.1 in a few ways. First, we replace the assumption that ZFC holds in $V$ with ZF plus the requirement that $G$ adds a set of ordinals to $V$ (which of course holds in the ZFC context, if $G \notin V$ ). Second, we only require that $M \subseteq V$, not that $M=V$ or $M$ is eventually stationarily correct or anything further, and so in particular, we allow $M$ to be illfounded. The proof also gives an alternate proof of Woodin's result, for the case that $G \notin V$; note that we do not use any stationary set combinatorics. The same kind of argument can be used in certain other places where the stationary set argument is traditionally used, for example, in Usuba's ZFC proof that the mantle coincides with the $\kappa$-mantle if $\kappa$ is extendible.

Lemma 2.2. Assume ZF. Let $G$ be $(V, \mathbb{P})$-generic for some forcing $\mathbb{P} \in V$ and let $\alpha \in \mathrm{OR}$. Let $\theta \in \mathrm{OR}$ be such that $V$ has no surjection $\mathbb{P}^{<\omega} \times \alpha \rightarrow \theta$. Suppose $(M, E, N, j)$ are such that $M, E \in V$ and $N, j \in V[G], N$ is transitive and rudimentarily closed, $\mathcal{H}_{\theta}^{V} \subseteq N$, and $j:(N, \in) \rightarrow(M, E)$ is elementary. (Note we allow $(M, E)$ to be illfounded.) Then:

1. $j\lceil\alpha \in V$,

2. $\mathcal{P}(\alpha) \cap N=\mathcal{P}(\alpha) \cap V$.

Proof. Part 2: This follows directly from part 1. For $\mathcal{P}(\alpha) \cap V \subseteq \mathcal{H}_{\theta}^{V} \subseteq N$ by assumption, and supposing $X \in N \cap \mathcal{P}(\alpha)$, for each $\xi<\alpha$, we have

$$
\xi \in X \Longleftrightarrow N \models " \xi \in X " \Longleftrightarrow(M, E) \models " j(\xi) \in j(X) ",
$$

but since $(M, E) \in V$ and $j\lceil\alpha \in V$ (by part 1), therefore $X \in V$.

Part 1: We prove a couple of claims.

Claim 1. $V[G] \models$ "there is no surjection $f: \mathbb{P}<\omega \times \alpha \rightarrow \theta$ ".

Proof. Suppose otherwise, and fix $\tilde{f} \in V$ with $\widetilde{f}_{G}=f$ and $p_{0} \in G$ such that $p_{0} \|_{\mathbb{P}}$ " $\widetilde{f}: \check{\mathbb{P}}<\omega \times \check{\alpha} \rightarrow \check{\theta}$ is surjective". We define a function $g \in V$. For $(p, \vec{q}, \dot{\xi}, \gamma)$ in $\mathbb{P} \times \mathbb{P}^{<\omega} \times \alpha \times \theta$, set

$$
g(p, \vec{q}, \xi)=\gamma \Longleftrightarrow p \leq p_{0} \text { and } p \models_{\mathbb{P}} \tilde{f}(\check{\vec{q}}, \check{\xi})=\check{\gamma}
$$

Note $A=\operatorname{dom}(g) \subseteq \mathbb{P} \times \mathbb{P}^{<\omega} \times \alpha$ and $g: A \rightarrow \theta$ is surjective, impossible.

In $V[G]$, we have $j\left\lceil\theta: \theta \rightarrow \mathrm{OR}^{M}\right.$. Fix $\widetilde{k} \in V$ with $\widetilde{k}_{G}=j \uparrow \theta$ and $p_{0} \in G$ with $p_{0} \models_{\mathbb{P}}$ " $\widetilde{k}: \check{\theta} \rightarrow \mathrm{OR}^{\check{M}} " .{ }^{4}$ Working in $V$, for $p \in \mathbb{P}$ with $p \leq p_{0}$, define $X_{p} \subseteq \theta$ and $j_{p}: X_{p} \rightarrow \mathrm{OR}^{M}$ the function giving all values of $\widetilde{k}$ decided by $p$; that is, for $\xi<\theta$,

$$
j_{p}(\xi)=x \Longleftrightarrow x \in \mathrm{OR}^{M} \text { and } p \models_{\mathbb{P}} \tilde{k}(\check{\xi})=\check{x},
$$

and $X_{p}=\operatorname{dom}\left(j_{p}\right)$. So $X_{p}, j_{p} \in V$.

\footnotetext{
${ }^{4}$ Here $\mathrm{OR}^{M}=\{x \in M \mid(M, E) \models$ " $x$ is an ordinal" $\}$, and $M$ might be illfounded.
} 
Claim 2. There is $p \in G$ such that $p \leq p_{0}$ and $X_{p}$ has ordertype $\beta>\alpha .^{5}$

Proof. We have $\theta=\bigcup_{p \in G, p<p_{0}} X_{p}$. Suppose the claim fails. In $V[G]$, define a surjection $f: \mathbb{P} \times \alpha \rightarrow \theta$ by setting $f(p, \xi)=$ the $\xi$ th element of $X_{p}$, in the case that $X_{p}$ is defined and has ordertype $>\xi$, and $f(p, \xi)=0$ otherwise. This contradicts Claim 1.

So fix $p$ as in Claim 2. Let $\alpha^{\prime}$ be the $\alpha$ th element of $X_{p}$. So $Y=X_{p} \cap \alpha^{\prime}$ has ordertype $\alpha$. Let $\pi: \alpha \rightarrow Y$ be the increasing enumeration of $Y$. Then $\alpha, Y, \pi \in \mathcal{H}_{\theta}^{V} \subseteq N$. Since $N$ is transitive,

$$
N \models " \pi: \alpha \rightarrow Y \text { is the increasing enumeration of } Y ",
$$

so applying $j$,

$$
(M, E) \models " j(\pi): j(\alpha) \rightarrow j(Y) \text { is the increasing enumeration of } j(Y) \text { ". }
$$

Let $j_{Y}=j\left\lceil Y \in V=j_{p}\left\lceil Y \in V\right.\right.$. Note that $j_{Y}(\pi(\xi))=j(\pi(\xi))=j(\pi)(j(\xi))$ for each $\xi<\alpha,{ }^{6}$ and $\operatorname{so}^{7} j\left\lceil\alpha=j(\pi)^{-1} \circ j_{Y} \circ \pi\right.$, and since $j_{Y}, \pi, j(\pi),(M, E) \in V$, we get $j\lceil\alpha \in V$.

From the lemma, we can deduce:

Theorem 2.3. Assume $(V, P) \models \mathrm{ZF}_{2}$. Let $G$ be $(V, \mathbb{P})$-generic for some $\mathbb{P} \in V$. Suppose there are $\alpha \in \mathrm{OR}$ and $X \subseteq \alpha$ with $X \in V[G] \backslash V$.

Then there is no $(j, M) \in P[G]$ with $M \subseteq V$ and $j: V[G] \rightarrow M$ elementary. Hence, there is no $j \in P[G]$ such that $j: V[G] \rightarrow V$ is elementary.

Question 2.4. Is it consistent that there is $j: V[G] \rightarrow M \subseteq V$ as in the statement of Theorem 2.3, if we instead have $\mathcal{P}(\alpha) \cap V[G] \subseteq V$ for each $\alpha \in$ OR? Does it follow that $V^{<\mathrm{OR}} \cap V[G] \subseteq V$ ? What about in the case that $M=V$ ?

What about the converse direction, i.e. an embedding $j: V \rightarrow V[G]$, where $G$ is set-generic over $V \models \mathrm{ZF}$ ?

In the next section we will see that if ZFR is consistent, then one can get $j: V \rightarrow M \subsetneq V$ where every set is (individually) set-generic over $M$; this fact should be compared with $[7, \S 4]$.

\section{The iterates of $(V, j)$}

\subsection{Basic properties}

Remark 3.1. As the reader familiar with inner model theory will see, the methods in this section are heavily based on analyses of HOD via direct limit systems of mice, and related methods from the study of Varsovian models. The present context actually provides a nice introduction to some of those ideas, which avoids various issues (iteration trees, fine structure, comparison).

\footnotetext{
${ }^{5}$ Note that the proof does not use AC.

${ }^{6}$ Here $j(\pi)(j(\xi))$ is a slight abuse of notation; it should really be $(j(\pi)(j(\xi)))^{M}$, i.e. the $x \in M$ such that $M \models$ " $x=j(\pi)(j(\xi))$ ".

7 The notation " $j(\pi)^{-1}$ " involves a similar abuse.
} 
We start with an easy observation. Assume $(V, j) \models \mathrm{ZFR}$ and let $\lambda=\kappa_{\omega}(j)$. Then for each $X \in V_{\lambda}$ (i) there is no cofinal $f: \omega \rightarrow \lambda$ with $f \in \mathrm{OD}_{X}$, and (ii) $j \uparrow \lambda \notin \mathrm{OD}_{X}$.

For suppose $f$ is $\mathrm{OD}_{X}$. Then there is in fact some such $f$ which is definable from $(X, \lambda)$, by minimizing on other ordinal parameters. Taking $n<\omega$ large enough, then $j^{n}(X)=X$, so $j^{n}(f)=f$, so $j^{n}(f(k))=f(k)$ for each $k<\omega$. But $\operatorname{rg}(f)$ is unbounded in $\lambda$, and $\lambda$ is the least fixed point of $j^{n}$ which is $>\operatorname{cr}\left(j^{n}\right)$, a contradiction. And from $j\left\lceil\lambda\right.$ we can define $\left\langle\kappa_{n}\right\rangle_{n<\omega}$, which we have just seen is not $\mathrm{OD}_{X}$, so $j \uparrow \lambda$ is not $\mathrm{OD}_{X}$.

Now in the study of $L\left(V_{\lambda+1}\right)$ under $I_{0}$, (see [20], for example), the iterates of $\left(L\left(V_{\lambda+1}\right), j\right)$ are an important focus. And Mohammad Golshani asked, on the discussion board Mathoverflow, what properties might hold of the intersection of the models $N_{\alpha}$ which we now define. ${ }^{8}$

Definition 3.2 (Iterates $\left.M_{\alpha}\right)$. Assume $(V, j) \models$ ZFR. Set $M_{0}=(V, j)$. We define, for $\alpha \leq \beta \in \mathrm{OR}$, the $\alpha$ th iterate $M_{\alpha}=\left(N_{\alpha}, j_{\alpha}\right)$, where $N_{\alpha}$ is a transitive proper class and $j_{\alpha}: N_{\alpha} \rightarrow N_{\alpha}$ a Reinhardt embedding of $N_{\alpha}$ (so $\left(N_{\alpha}, j_{\alpha}\right) \models$ ZFR) and $i_{\alpha \beta}: M_{\alpha} \rightarrow M_{\beta}$ is elementary (literally a class function $N_{\alpha} \rightarrow N_{\beta}$, but elementary with respect to the predicates $\left.j_{\alpha}, j_{\beta}\right)$, and such that $i_{\alpha \gamma}=i_{\beta \gamma} \circ i_{\alpha \beta}$ for all $\alpha \leq \beta \leq \gamma$, and $i_{\alpha \alpha}=\mathrm{id}$, as follows. Given $M_{\beta}$, set $M_{\beta+1}=\left(N_{\beta}, j_{\beta}\left(j_{\beta}\right)\right)$ and $i_{\alpha, \beta+1}=j_{\beta} \circ i_{\alpha \beta}$. Given $M_{\beta}$ for all $\beta<\eta$ where $\eta$ is a limit, set $M_{\eta}$ as the direct limit, and $i_{\alpha \eta}$ the direct limit map.

By elementarity, $N_{\alpha}$ is extensional. Assuming that $N_{\alpha}$ is wellfounded, we identify it with its transitive collapse.

We introduce some symbols to the ZFR language to express these notions in formal language. For example, $\widetilde{M}$ represents the function $\alpha \mapsto M_{\alpha}$ defined under ZFR as above. So with the models above and $\alpha \in \mathrm{OR}^{V}$, we have $\left(\widetilde{M}_{\alpha}\right)^{(V, j)}=$ $M_{\alpha}$, and if $M_{\alpha}$ is wellfounded then $\left(\widetilde{M}_{\beta}\right)^{M_{\alpha}}=M_{\alpha+\beta}$. We write $\widetilde{N}_{\alpha}, \widetilde{j}_{\alpha}, \widetilde{i}_{\alpha \beta}$ similarly. (This literally overloads the symbol $\widetilde{j}$ with two interpretations, but when we write $\widetilde{j}$ without a subscript, it always refers to the original function $\left.\widetilde{j}_{0} \cdot\right)$

We now work in a model $(V, j) \models$ ZFR.

As pointed out by Hamkins on Mathoverflow (see Footnote 8), the usual proof of linear iterability with respect to a single normal measure gives the following (iterability also holds in the $I_{0}$ case and generalizations thereof, but the proof is finer there; see [20] and [15]):

Lemma 3.3. For all $\alpha \in \mathrm{OR}, M_{\alpha}$ is a wellfounded (we take it transitive), $M_{\alpha}=\left(N_{\alpha}, j_{\alpha}\right) \models \mathrm{ZFR}$, and $i_{\beta \alpha}: M_{\alpha} \rightarrow M_{\beta}$ is elementary and $\in$-cofinal. ${ }^{9}$

Proof. Everything is proved by induction. We get the elementarity because $M_{0}=\left(N_{0}, j_{0}\right) \models \mathrm{ZFR}$, so for each $n<\omega$ there is a club class of $\alpha$ such that $\left(V_{\alpha}^{N_{0}}, j\left\lceil V_{\alpha}^{N_{0}}\right) \preccurlyeq_{n}\left(N_{0}, j\right)\right.$, which easily gives that $i_{01}$ is elementary with respect to $M_{0}, M_{1}$, not just $N_{0}, N_{1}$, and likewise for larger indices.

\footnotetext{
${ }^{8}$ For the discussion see https://mathoverflow.net/questions/185253/reinhardt-cardinalsand-iterability. Note there is a slight difference in notation; we are writing $N_{\alpha}$ for what he refers to as $M_{\alpha}$ there.

${ }^{9}$ Note that the elementarity is with respect to $M_{\alpha}=\left(N_{\alpha}, j_{\alpha}\right)$ and $M_{\beta}=\left(N_{\beta}, j_{\beta}\right)$, not just $N_{\alpha}, N_{\beta}$.
} 
Now suppose that $\eta \in \mathrm{OR}$ and $M_{\eta}$ is illfounded, and $\eta$ is least such. Then $\mathrm{OR}^{M_{\eta}}$ is illfounded (consider ranks of elements). Since $M_{\alpha+1}$ and $M_{\alpha}$ have the same universe, $\eta$ is a limit ordinal. Let $\lambda \in \mathrm{OR}$ be least with $i_{0 \eta}(\lambda)$ in the illfounded part of $\mathrm{OR}^{M_{\eta}}$. There is $\alpha<\eta$ and $\lambda_{1} \in \mathrm{OR}$ such that $\lambda^{\prime}=i_{0 \alpha}(\lambda)>$ $\lambda_{1}$ and $i_{\alpha \eta}\left(\lambda_{1}\right)$ is in the illfounded part of $M_{\eta}$. Let $\eta^{\prime}=i_{0 \alpha}(\eta) \geq \eta$. By elementarity of $i_{0 \alpha}, M_{\alpha} \models$ " $\eta^{\prime}$ is the least $\gamma \in \mathrm{OR}$ such that $\widetilde{M}_{\gamma}$ is illfounded". Since $M_{\alpha}$ is wellfounded, $\left(\widetilde{M}_{\beta}\right)^{M_{\alpha}}=M_{\alpha+\beta}$ and $\alpha+\eta^{\prime}=\eta$, so $\eta^{\prime}=\eta$ and $\widetilde{M}_{\eta}^{M_{\alpha}}=M_{\eta}$. By elementarity, $M_{\alpha} \models$ " $\lambda^{\prime}$ is the least $\xi \in \mathrm{OR}$ with $\widetilde{i}_{0 \eta}(\xi)$ in the illfounded part of $\widetilde{M}_{\eta} "$. But $\widetilde{i}_{0 \eta}^{M_{\alpha}}=i_{\alpha \eta}$, and we chose $\alpha$ with $i_{\alpha \eta}\left(\lambda_{1}\right)$ in the illfounded part of $M_{\eta}$, a contradiction.

Lemma 3.4. Let $x \in M_{\omega}$ and $m<\omega$ with $x \in \operatorname{rg}\left(i_{m \omega}\right)$. Then $i_{m n}(x)=x$ for all $n \in[m, \omega)$.

Proof. Let $\bar{x}$ be such that $i_{m \omega}(\bar{x})=x$. Then $M_{m} \models " x=\widetilde{i}_{0 \omega}(\bar{x})$ ", so letting $\left(\bar{x}^{\prime}, x^{\prime}\right)=i_{m n}(\bar{x}, x)$, elementarity gives $M_{n} \models$ " $x^{\prime}=\widetilde{i}_{0 \omega}\left(\bar{x}^{\prime}\right)$ ", but $\widetilde{i}_{0 \omega}^{M_{n}}=i_{n \omega}$, so by commutativity, $x^{\prime}=i_{m \omega}(\bar{x})=x$.

By the lemma, the following definition makes sense:

Definition 3.5. For $x \in M_{\omega}$ let $x^{*}=\lim _{n<\omega} i_{n \omega}(x)=i_{m \omega}(x)$ for any $/$ all $m<\omega$ with $x \in \operatorname{rg}\left(i_{m \omega}\right)$.

Lemma 3.6. For $x \in M_{\omega}$, we have $x^{*}=i_{\omega, \omega+\omega}(x)={\widetilde{i_{0 \omega}}}^{M_{\omega}}(x)$.

Proof. Given $x$, let $\bar{x}$ and $m<\omega$ with $i_{m \omega}(\bar{x})=x$. Then $M_{m} \models " \widetilde{i}_{0 \omega}(\bar{x})=x "$, so since $x^{*}=i_{m \omega}(x)$, by elementarity we have $M_{\omega} \models \widetilde{i}_{0 \omega}(x)=x^{*}$ ", but $\widetilde{i}_{0 \omega}^{M_{\omega}}=$ $i_{\omega, \omega+\omega}$, so $i_{\omega, \omega+\omega}(x)=x^{*}$.

We now establish that $N_{\omega}$ is closed under definability in $V$. Note that the notation $\operatorname{Hull}^{\left(V, M_{\omega}\right)}(X)$ refers to definability in $V$ with $M_{\omega}$ as a predicate, from parameters in $X$.

Theorem 3.7. Let $\kappa_{n}=\operatorname{cr}\left(j_{n}\right)$ and $\lambda=\kappa_{\omega}(j)$. Then:

1. $\operatorname{HOD} \subseteq \operatorname{Hull}^{V}\left(N_{\omega}\right)=\operatorname{Hull}^{\left(V, M_{\omega}\right)}\left(N_{\omega}\right)=N_{\omega}$,

2. $V_{\lambda}^{\mathrm{HOD}}=V_{\lambda}^{\mathrm{HOD}^{N_{\omega}}}$ and $V_{\lambda+1}^{\mathrm{HOD}} \subseteq V_{\lambda+1}^{\mathrm{HOD}^{N_{\omega}}}$.

3. $\lambda$ is greatly Mahlo in $\mathrm{HOD}$ and weakly compact in $\mathrm{HOD}^{N_{\omega}}$.

4. $\lambda$ is $N_{\omega}$-Berkeley in $V .^{10}$

5. $\left(\lambda^{+}\right)^{\mathrm{HOD}} \leq\left(\lambda^{+}\right)^{N_{\omega}}<\lambda^{+}$

6. If $\kappa_{0}^{+}$is regular or $\operatorname{cof}\left(\kappa_{0}^{+}\right)=\kappa_{0}$ then $\operatorname{cof}\left(\left(\lambda^{+}\right)^{M_{\omega}}\right)=\omega$.

7. If $\operatorname{cof}\left(\kappa_{0}^{+}\right)<\kappa_{0}$ then $\operatorname{cof}\left(\left(\lambda^{+}\right)^{M_{\omega}}\right)=\operatorname{cof}\left(\kappa_{0}^{+}\right)$.

\footnotetext{
${ }^{10}$ Note this generalizes the (folklore?) fact that $\lambda$ is HOD-Berkeley, mentioned in $\left[6, \S^{* * *}\right]$.
} 
Proof. Part 1: We just need to see that $\operatorname{Hull}^{\left(V, M_{\omega}\right)}\left(N_{\omega}\right) \subseteq N_{\omega}$. We prove this by induction on rank of elements of $\operatorname{Hull}^{\left(V, M_{\omega}\right)}\left(N_{\omega}\right)$. So let $X \in \operatorname{Hull}^{\left(V, M_{\omega}\right)}\left(N_{\omega}\right)$ with $X \subseteq N_{\omega}$; we must see that $X \in N_{\omega}$. Let $\varphi$ be a formula in $\mathcal{L}_{\dot{M}}$ and $p \in N_{\omega}$ be such that for $x \in N_{\omega}$, we have $x \in X$ iff $V \models \varphi\left(x, p ; M_{\omega}\right)$, with $M_{\omega}$ interpreting the predicate symbol $\dot{M}$. Then for $n<\omega, x \in X$ iff $M_{n} \models$ $\varphi\left(x, p ; \widetilde{M}_{\omega}\right)$ iff $M_{\omega} \models \varphi\left(x^{*}, p^{*} ; \widetilde{M}_{\omega}\right)$ iff $M_{\omega} \models \varphi\left(\widetilde{i}_{0 \omega}(x), \widetilde{i}_{0 \omega}(p), \widetilde{M}_{\omega}\right)$, so $X$ is computed by $M_{\omega}$, so $X \in N_{\omega}$, as desired.

Part 2: Because $\lim _{n<\omega} \operatorname{cr}\left(i_{n \omega}\right)=\lambda$, we easily have $V_{\lambda}^{\mathrm{HOD}}=V_{\lambda}^{\mathrm{HOD}^{N_{\omega}}}$. If $A \in V_{\lambda+1}^{\mathrm{HOD}}$ then just note that $A^{*} \in \mathrm{HOD}^{N_{\omega}}$ and $A^{*} \cap V_{\lambda}=A$, so $A \in V_{\lambda+1}^{\mathrm{HOD}^{N_{\omega}}}$.

Part 3: Since $\lambda=\operatorname{cr}\left(j_{\omega}\right)$ and $j_{\omega}\left\lceil\mathrm{HOD}^{N_{\omega}}: \mathrm{HOD}^{N_{\omega}} \rightarrow \mathrm{HOD}^{N_{\omega}}\right.$ is elementary, we easily get that $\lambda$ is weakly compact in $\operatorname{HOD}^{N_{\omega}}$, hence greatly Mahlo in $\operatorname{HOD}^{N_{\omega}}$, which by part 2 implies $\lambda$ is greatly Mahlo in HOD.

Part 4: By Lemma 3.4, for every $M \in N_{\omega}$, there is $n<\omega$ such that $j_{m}(M)=$ $M$ for all $m \in[n, \omega)$, and since $\lambda=\sup _{m<\omega} \operatorname{cr}\left(j_{m}\right)$, this suffices.

Parts 5-7: These facts now follow easily by considering the direct limit producing $\left(\lambda^{+}\right)^{M_{\omega}}$. We have $\left(\lambda^{+}\right)^{M_{\omega}}<\lambda^{+}$because there is a surjection from $\omega \times \lambda \rightarrow\left(\lambda^{+}\right)^{M_{\omega}}$ computable directly from the direct limit. If $\kappa_{0}^{+}$is regular or $\operatorname{cof}\left(\kappa_{0}^{+}\right)=\kappa_{0}$ then $j_{m n}$ (where $m \leq n<\omega$ ) is discontinuous at $\kappa_{m}^{+}$, and since $\left(\lambda^{+}\right)^{M_{\omega}}$ is the direct limit of an $\omega$-sequence of these, this ordinal has cofinality $\omega$. In the other case, $j_{m n}$ and $j_{m \omega}$ is continuous at $\left(\kappa_{0}^{+}\right)$.

Question 3.8. Is/can we have $V_{\lambda+1}^{\mathrm{HOD}}=V_{\lambda+1}^{\mathrm{HOD}^{N_{\omega}}}$ ? Or $V_{\lambda+1}^{\mathrm{HOD}}=V_{\lambda+1}^{N_{\omega}}$ ? Is $\lambda$ Woodin in HOD? Weakly compact in HOD? Reflecting in HOD? ${ }^{11}$ These properties seem (to the author) reasonable to ask about, since $\lambda$ has them in $M_{\omega}$ and $\mathrm{HOD} \subseteq M_{\omega}$. If $V_{\lambda} \subseteq$ HOD then $\lambda$ is clearly Woodin in HOD, since it is Woodin (and more) in $N_{\omega}$.

\subsection{Every set is generic over $N_{\alpha}$}

By Vopenka, every set is set-generic over HOD. Moreover:

Lemma 3.9. Every set in $V$ is set-generic over $M_{\omega}$.

Proof. One way to prove this is to use a Vopenka argument, as done in [15]. But we want to generalize the theorem later to $M_{\alpha}$ for arbitrary $\alpha$, and there we will not have HOD $\subseteq M_{\alpha}$. So we use a different argument, which seems to be more generalizable.

We will show directly that for each $\beta \in \mathrm{OR}$ and each $X \subseteq V_{\beta}^{M_{\omega}}$, with $X \in V$, we can add $X$ set-generically to $M_{\omega}$. This suffices, because letting $\alpha \in \mathrm{OR}$, we can apply this to $X=i_{0 \omega}$ " $V_{\alpha}$, and then $V_{\alpha}^{M_{\omega}[G]}=V_{\alpha}$ (and $G$ adds $j\left\lceil V_{\alpha}\right.$ also).

The forcing $\mathbb{P}$ we use is the natural adaptation of Schindler's forcing (to appear in [13]) to the current setting (and Schindler's forcing is related to the forcings of Vopenka, Bukowsky and Woodin's extender algebra; cf. 3.23).

Work in $M_{\omega}$. Let $\mathcal{L}$ be the class of all infinitary Boolean formulas $\varphi$ in propositional symbols $P_{x}$, for each $x \in V_{\beta}$. That is, $\mathcal{L}$ is the minimal class generated by the following rules:

1. For each $x \in V_{\beta}$, we have a corresponding propositional symbol $P_{x} \in \mathcal{L}$ (with $x \neq y \Longrightarrow P_{x} \neq P_{y}$ ).

\footnotetext{
${ }^{11}$ Recall that $\kappa$ is a reflecting cardinal iff $\kappa$ is inaccessible and for every stationary $A \subseteq \kappa$ there are stationarily many regular $\bar{\kappa}<\kappa$ such that $A \cap \bar{\kappa}$ is stationary in $\bar{\kappa}$.
} 
2. If $\varphi \in \mathcal{L}$ then $\neg \varphi \in \mathcal{L}$.

3. If $A$ is a set and $A \subseteq \mathcal{L}$ then $\bigvee A \in \mathcal{L}$ and $\bigwedge A \in \mathcal{L}$.

Working for a moment in a possibly larger universe, given $\varphi \in \mathcal{L}$ and a set $X \subseteq V_{\beta}^{M_{\omega}}$, we define satisfaction $X \models \varphi$ recursively in the obvious manner: $X \models P_{x}$ iff $x \in X, X \models \neg \varphi$ iff $\neg(X \models \varphi), X \models \bigvee A$ iff $X \models \varphi$ for some $\varphi \in A$, and $X \models \bigwedge A$ iff $X \models \varphi$ for all $\varphi \in A$.

Recall $x^{*}=i_{\omega, \omega+\omega}(x)=\widetilde{i}_{0 \omega}^{M_{\omega}}(x)$ in $M_{0}$. Work again in $M_{\omega}$. For $\varphi \in \mathcal{L}$, let

$$
\mathscr{E}_{\varphi}=\left\{X \subseteq V_{\beta^{*}} \mid X \models \varphi^{*}\right\} .
$$

Let $\mathcal{L}^{\prime}$ be the class of formulas $\varphi \in \mathcal{L}$ such that $\mathscr{E}_{\varphi} \neq \emptyset$. Given $\varphi, \psi \in \mathcal{L}^{\prime}$, set $\varphi \equiv \psi$ iff $\mathscr{E}_{\varphi}=\mathscr{E}_{\psi}$. So there are only set-many (in fact at most $V_{\beta^{*}+2^{-}}$ many) equivalence classes. Let $\mathbb{P}$ be the partial order whose conditions are the equivalence classes $[\varphi]$, and with $[\varphi] \leq[\psi] \Longleftrightarrow \mathscr{E}_{\varphi} \subseteq \mathscr{E}_{\psi}$. This is a partial order (forcing equivalent to a partial order $\subseteq V_{\beta^{*}+2}$ ).

Now work in $V$ and let $X \subseteq V_{\beta}$. Let

$$
G_{X}=\left\{[\varphi] \in \mathbb{P} \mid \varphi \in \mathcal{L}^{\prime} \text { and } X \models \varphi\right\} .
$$

Here if $\varphi, \psi \in \mathcal{L}^{\prime}$ and $\varphi \equiv \psi$ then $X \models \varphi \Leftrightarrow X \models \psi$. For suppose that $X \models \varphi \wedge \neg \psi$. Let $n<\omega$ be large enough that $i_{n m}(\varphi \wedge \neg \psi)=\varphi \wedge \neg \psi$ and $i_{n m}(\beta)=\beta$ for all $m \in[n, \omega)$. Then note that

$$
V \models \text { "There is } X^{\prime} \subseteq V_{\beta} \text { such that } X^{\prime} \models \varphi \wedge \neg \psi \text { " }
$$

(as witnessed by $X$ ). Therefore, applying $i_{n \omega}$, we have

$$
M_{\omega} \models \text { "There is } X^{\prime} \subseteq V_{\beta^{*}} \text { such that } X^{\prime} \models(\varphi \wedge \neg \psi)^{*} \text { ". }
$$

But then for any such $X^{\prime}$, we have $X^{\prime} \models \varphi^{*}$ and $\neg\left(X^{\prime} \models \psi^{*}\right)\left(\right.$ as $(\varphi \wedge \neg \psi)^{*}=$ $\left.\varphi^{*} \wedge \neg\left(\psi^{*}\right)\right)$, so $\mathscr{E}_{\varphi} \neq \mathscr{E}_{\psi}$, a contradiction.

Now we claim that $G_{X}$ is $\left(M_{\omega}, \mathbb{P}\right)$-generic; and then it is easy to see that $M_{\omega}\left[G_{X}\right]=M_{\omega}[X]$. For $G_{X}$ is easily a filter, so we just need to verify genericity. So let $D \in \mathcal{P}(\mathbb{P}) \cap M_{\omega}$ be dense. We must see that $G_{X} \cap D \neq \emptyset$. Note that since $D$ is a set, for each $\xi \in \mathrm{OR}, M_{\omega}$ has the set

$$
E=\left\{\varphi \in \mathcal{L}^{\prime} \cap V_{\xi} \mid[\varphi] \in D\right\}
$$

and taking $\xi$ large enough, $D=\{[\varphi] \mid \varphi \in E\}$. Now $\bigvee E \in \mathcal{L}$. We must see that $X \models \bigvee E$, because then $X \models \varphi$ for some $\varphi \in E$, and therefore $G_{X} \cap D \neq \emptyset$.

So suppose $X \models \neg \bigvee E$. Let $n<\omega$ with $i_{n, n+1}(E, \beta)=(E, \beta)$. Then

$$
\begin{gathered}
V \models \text { "There is } X^{\prime} \subseteq V_{\beta} \text { such that } X^{\prime} \models \neg \bigvee E \text { ", } \\
M_{\omega} \models \text { "There is } X^{\prime} \subseteq V_{\beta^{*}} \text { such that } X^{\prime} \models(\neg \bigvee E)^{*} \text { ". }
\end{gathered}
$$

So $\neg \bigvee E \in \mathcal{L}^{\prime}$. By the density of $D$, there is $\varphi \in E$ such that $[\varphi]$ is compatible with $[\neg \bigvee E]$. So let $\psi \in \mathcal{L}^{\prime}$ with $[\psi] \leq[\varphi]$ and $[\psi] \leq[\neg \bigvee E]$. Then $\emptyset \neq \mathscr{E}_{\psi} \subseteq$ $\mathscr{E}_{\varphi} \cap \mathscr{E}_{\neg \bigvee E}$. So let $Y \in M_{\omega}$, with $Y \subseteq V_{\beta^{*}}$, witness that $\mathscr{E}_{\varphi} \cap \mathscr{E}_{\neg \bigvee E} \neq \emptyset$. Then $Y \models \varphi^{*}$ and $Y \models(\neg \bigvee E)^{*}$, but note $(\neg \bigvee E)^{*}=\neg \bigvee\left(E^{*}\right) \equiv \bigwedge\left\{\neg \varrho \mid \varrho \in E^{*}\right\}$ and $\varphi \in E$, so $\varphi^{*} \in E^{*}$, so $Y \models \varphi^{*}$ and $Y \models \neg \varphi^{*}$, contradiction. 
We can immediately deduce:

Corollary 3.10. Suppose $(V, j) \models$ ZFR. Let $\alpha<\omega^{2}$. Then every set in $V$ is set-generic over $M_{\alpha}$.

Proof. We have this for $\alpha<\omega+\omega$, by Theorem 3.9. Consider then $\alpha=\omega+\omega$. By Theorem 3.9, applied in $M_{\omega}$, we get that every set in $M_{\omega}$ is set-generic over $M_{\omega+\omega}$. Therefore given any $\alpha \in \mathrm{OR}$, we can find a set-generic extension $M_{\omega+\omega}[G]$ of $M_{\omega+\omega}$ such that $V_{\alpha}^{M_{\omega+\omega}[G]}=V_{\alpha}^{M_{\omega}}$. But then for any set $X \in V$, by taking $\alpha$ high enough, we get that $X$ is set-generic over $M_{\omega+\omega}[G]$. So we have added $X$ by a 2 -step iteration over $M_{\omega+\omega}$, which suffices. Clearly this generalizes to all $\alpha<\omega+\omega$.

We next generalize these initial facts to arbitrary iterates.

Definition 3.11. For a limit $\alpha$, say $x$ is $\alpha$-eventually stable $(\alpha$-es) iff $x \in$ $\bigcap_{\xi<\alpha} M_{\xi}$ and there is $\xi<\alpha$ such that $i_{\xi \gamma}(x)=x$ for all $\gamma \in[\xi, \alpha)$. Say that $x$ is hereditarily $\alpha$-eventually stable ( $\alpha$-hes) iff every $y \in \operatorname{trancl}(\{x\})$ is $\alpha$-es. For $\alpha$-es $x$, let $x_{\alpha}^{*}=\lim _{\xi<\alpha} i_{\xi \alpha}(x)$. Note here that $i_{\xi \alpha}(x)=i_{\gamma \alpha}(x)$ whenever $\xi \leq \gamma<\alpha$ and $\xi$ witnesses the $\alpha$-eventual stability of $x$.

Definition 3.12. Given an ordinal $\alpha>0$, the eventual ordertype $\operatorname{eot}(\alpha)$ of $\alpha$ is the least $\chi$ such that $\alpha=\xi+\chi$ for some $\xi<\alpha$.

Note that (i) $\alpha$ is a successor iff $\operatorname{eot}(\alpha)=1$ iff $\operatorname{eot}(\alpha)$ is a successor; (ii) $\operatorname{eot}(\alpha)=\operatorname{eot}(\operatorname{eot}(\alpha))$; (iii) $\alpha=\xi+\operatorname{eot}(\alpha)$ for all sufficiently large $\xi<\alpha$. Clearly $\eta=\operatorname{eot}(\alpha) \leq \alpha \leq \kappa_{\alpha}$, and if $\eta<\kappa_{\alpha}$ then $\eta_{\alpha}^{*}=\eta$, whereas if $\eta=\alpha=\kappa_{\alpha}$ then $\alpha<\alpha_{\alpha}^{*}=\kappa_{\alpha_{\alpha}^{*}}$ :

Lemma 3.13. Suppose $(V, j) \models \mathrm{ZFR}$. Let $\alpha$ be a limit ordinal. Then:

1. $N_{\alpha}=\{x \mid x$ is $\alpha$-hes $\}$

2. $x_{\alpha}^{*}=i_{\alpha, \alpha+\eta_{\alpha}^{*}}(x)=\widetilde{i}_{0 \eta_{\alpha}^{*}}^{M_{\alpha}}(x)$ where $\eta=\operatorname{eot}(\alpha)$.

Proof. First note that all ordinals are $\alpha$-es, since $M_{\alpha}$ is wellfounded.

Now let $x \in M_{\alpha}$; we show that $x$ is $\alpha$-hes. We have $M_{\alpha} \subseteq M_{\xi}$ for each $\xi<\alpha$. Assume by induction that every $y \in \operatorname{trancl}(x)$ is $\alpha$-es. So we need to see $x$ is $\alpha$-es. Let $\xi<\alpha$ be such that $x \in \operatorname{rg}\left(i_{\xi \alpha}\right)$ and $\xi+\eta=\alpha$ where $\eta=\operatorname{eot}(\alpha)$, and $i_{\xi \gamma}(\eta)=\eta$ for all $\gamma \in[\xi, \alpha)$. We claim that $i_{\xi \gamma}(x)=x$ for all $\gamma \in[\xi, \alpha)$. For $x=i_{\xi \alpha}(\bar{x})=\widetilde{i}_{0 \eta}^{M_{\xi}}(\bar{x})$ for some $\bar{x}$, so $i_{\xi \gamma}(x)=i_{\xi \gamma}\left(\widetilde{i}_{0 \eta}^{M_{\xi}}\right)\left(i_{\xi \gamma}(\bar{x})\right)=\widetilde{i}_{0 \eta}^{M_{\gamma}}\left(i_{\xi \gamma}(\bar{x})\right)$, but $\gamma+\eta=\alpha$, so $\widetilde{i}_{0 \eta}^{M_{\gamma}}\left(i_{\xi \gamma}(\bar{x})\right)=i_{\gamma \alpha}\left(i_{\xi \gamma}(\bar{x})\right)=i_{\xi \alpha}(\bar{x})=x$, as desired.

Now let $x \in M_{\alpha}$ and $\eta=\operatorname{eot}(\alpha)$. We must see that $x_{\alpha}^{*}=i_{\alpha, \alpha+\eta_{\alpha}^{*}}(x)$. Let $\xi<\alpha$ be such that (i) $\xi+\eta=\alpha$, (ii) $x \in \operatorname{rg}\left(i_{\xi \alpha}\right)$, and (iii) $i_{\xi \gamma}(x, \eta)=(x, \eta)$ for all $\gamma \in[\xi, \alpha)$. Then $\left(x_{\alpha}^{*}, \eta_{\alpha}^{*}\right)=i_{\xi \alpha}(x, \eta)$; let $\bar{x}$ be such that $i_{\xi \alpha}(\bar{x})=x$. Since $M_{\xi} \models " x=\widetilde{i}_{0 \eta}(\bar{x})$ ", applying $i_{\xi \alpha}$, we get

$$
M_{\alpha} \models " x_{\alpha}^{*}=\widetilde{i}_{0 \eta_{\alpha}^{*}}(x) ",
$$

so $i_{\alpha, \alpha+\eta_{\alpha}^{*}}(x)=x_{\alpha}^{*}$, as desired.

Theorem 3.14. Suppose $(V, j) \models \mathrm{ZFR}$ and let $\tau \in \mathrm{OR}$. Then every set in $V$ is set-generic over $M_{\tau}$. 
Proof. By induction on $\tau$. Successor steps are trivial, so let $\tau$ be a limit and $\chi=\operatorname{eot}(\tau)$. We write $x^{*}=x_{\tau}^{*}=i_{\tau, \chi_{\tau}^{*}}(x)$ throughout (as computed in $(V, j)$ ).

Let $\alpha \in \mathrm{OR}$ and $\beta=\sup i_{0 \tau}$ " $\alpha$. Let $X=i_{0 \tau}$ " $V_{\alpha}$. It suffices to see that $X$ is set-generic over $M_{\tau}$. We will just use the fact that $X \subseteq V_{\beta}^{M_{\tau}}$.

Now $X$ is set-generic over $M_{\xi}$ for each $\xi<\tau$. Fix $\delta \in \mathrm{OR}$ such that for each $\xi<\tau$, there is an $\left(M_{\xi}, \operatorname{Col}\left(\omega, V_{\delta}^{M_{\xi}}\right)\right)$-generic $G$ such that $X \in M_{\xi}[G]$.

Work in $M_{\tau}$. Let $\mathcal{L}$ be as before (with $P_{x}$ for each $x \in V_{\beta}$ ). Let $\mathbb{Q}=$ $\operatorname{Col}\left(\omega, V_{\delta^{*}}\right)$. For $\varphi \in \mathcal{L}$, let $\mathscr{E}_{\varphi}$ be the $\mathbb{Q}$-name for the set of all $X \in \mathcal{P}\left(V_{\beta^{*}}^{\widetilde{M}_{\chi^{*}}}\right) \cap$ $V^{\mathbb{Q}}$ such that $X \models \varphi^{*}$. Let $\mathcal{L}^{\prime}$ be the class of all $\varphi \in \mathcal{L}$ such that $\mathbb{Q} \Vdash \mathscr{E}_{\varphi} \neq \emptyset$ (equivalently, there is $p \in \mathbb{Q}$ such that $p \| \mathscr{E}_{\varphi} \neq \emptyset$ ). Given $\varphi, \psi \in \mathcal{L}^{\prime}$, let $\varphi \equiv \psi$ iff $\mathbb{Q} \| \mathscr{E}_{\varphi}=\mathscr{E}_{\psi}$. Let $[\varphi]$ be the equivalence class of $\varphi \in \mathcal{L}^{\prime}$. Let $\mathbb{P}$ be the partial order whose conditions are the equivalence classes $[\varphi]$, with $[\varphi] \leq[\psi]$ iff $\mathbb{Q} \Vdash \mathscr{E}_{\varphi} \subseteq \mathscr{E}_{\psi}$.

Now work in $V$. Let $G_{X}=\left\{\varphi \in \mathcal{L}^{\prime} \mid X \models \varphi\right\}$. We claim that $G$ is $\left(M_{\tau}, \mathbb{P}\right)$-generic; it follows that $M_{\tau}\left[G_{X}\right]=M_{\tau}[X]$. This is proven like before. Let $D \in \mathcal{P}(\mathbb{P}) \cap M_{\tau}$ be dense. Let $E \in M_{\tau}$ be a such that $D=\{[\varphi] \mid \varphi \in E\}$. We need to see that $X \models \bigvee E$, so suppose otherwise. Then for all sufficiently large $\xi<\tau$,

$$
M_{\xi} \models \text { let } Y=V_{\beta}^{\widetilde{M}_{\chi}} \text {; then } \operatorname{Col}\left(\omega, V_{\delta}\right) \Vdash \exists X^{\prime} \subseteq \check{Y} \text { such that } X^{\prime} \models \neg \bigvee E \text {, }
$$

(as witnessed by $X$, by induction and choice of $\delta$ ), which gives that

$$
M_{\tau} \models \text { "let } Y=V_{\beta^{*}}^{\widetilde{M}_{\chi^{*}}} \text {; then } \mathbb{Q} \Vdash \exists X^{\prime} \subseteq \check{Y} \text { such that } X^{\prime} \models(\neg \bigvee E)^{*} \text {. }
$$

So $(\neg \bigvee E) \in \mathcal{L}^{\prime}$, so $[\neg \bigvee E] \in \mathbb{P}$.

Work in $M_{\tau}$. By the density of $D$ and since $[\neg \bigvee E] \in \mathbb{P}$, there is $\varphi \in E$ such that $[\varphi]$ is compatible with $[\neg \bigvee E]$. Let $[\psi] \in \mathbb{P}$ with $[\psi] \leq[\varphi]$ and $[\psi] \leq[\neg \bigvee E]$. Let $G$ be $\operatorname{Col}\left(\omega, V_{\delta^{*}}\right)$-generic. Then in $V[G]$, there is $X^{\prime}$ such that $X^{\prime} \models \psi^{*}$, and by the definition of $\leq$, therefore $X^{\prime} \models \varphi^{*}$ and $X^{\prime} \models(\neg \bigvee E)^{*}$, so $X^{\prime} \models \bigwedge_{\varrho \in E^{*}} \neg \varrho$, so $X^{\prime} \models \neg \varphi^{*}$, a contradiction.

We deduce the existence of some interesting forcing extensions under ZFR. Recall the notion of virtual large cardinals from [3].

Definition 3.15. $\delta$ is virtually Berkeley iff for every transitive $M$ with $\delta \in M$ and every $\eta<\delta$, there is a forcing $\mathbb{P}$ which forces the existence of an elementary $j: M \rightarrow M$ with $\eta<\operatorname{cr}(j)<\delta$. Say that $\delta$ is $V_{\alpha}$-preserving-virtually Berkeley iff $\delta$ is virtually Berkeley, as witnessed by forcings $\mathbb{P}$ which preserve $V_{\alpha}$.

Corollary 3.16. Suppose $(V, j) \models$ ZFR. Let $\kappa=\operatorname{cr}(j)$ and $\lambda=\kappa_{\omega}(j)$. Then there are partial orders $\mathbb{P}_{0}, \mathbb{P}_{1} \in V$ such that:

1. $\kappa$ is $V_{\kappa}$-preserving-virtually Berkeley.

2. $\mathbb{P}_{0} \Vdash V_{\breve{\kappa}}=\check{V}_{\kappa}$ and there is an elementary $k: V_{\breve{\kappa}} \rightarrow V_{\breve{\kappa}}$ such that

(a) $\kappa_{\omega}(k)=\check{\kappa}($ hence $\operatorname{cof}(\check{\kappa})=\omega)$ and

(b) $\left(\check{V}_{\lambda}, \breve{j}\left\lceil\check{V}_{\lambda}\right)\right.$ is the $\omega$ th iterate of $\left(\check{V}_{\kappa}, k\right)$. 
3. for each $\beta<\kappa$ there is $\bar{\lambda} \in(\beta, \kappa)$ such that $\mathbb{P}_{1}$ forces that $V_{\check{\bar{\lambda}}}=\check{V}_{\bar{\lambda}}$ and $\left(\bar{\lambda}^{+}\right)=\check{\kappa}$ and there is a rank-into-rank embedding $k: V_{\overline{\bar{\lambda}}} \rightarrow V_{\overline{\bar{\lambda}}}$ such that $\left(\check{V}_{\lambda}, \check{j}\left\lceil\check{V}_{\lambda}\right)\right.$ is the $\check{\kappa}$ th iterate of $\left(V_{\check{\bar{\lambda}}}, k\right)$.

Proof. The corresponding things are forceable over $M_{\omega}$ (for parts 1 and 2) and over $M_{\left(\lambda^{+}\right)}$(for part 3) with respect to $\lambda$, respectively. So the elementarity of $i_{0 \omega}$ and $i_{0\left(\lambda^{+}\right)}$pulls them back to $M_{0}=(V, j)$.

\subsection{Intersections of iterates}

We consider now intersections of the form $\bigcap_{\xi<\tau} N_{\xi}$.

Definition 3.17. Suppose $(V, j) \models$ ZFR and let $\tau$ be a limit ordinal and $\beta<\tau$. Let $x \in \bigcap_{\xi<\tau} N_{\xi}$. Then $x$ is $(\beta, \tau)$-stable iff $i_{\beta \gamma}(x)=x$ for all $\gamma \in[\beta, \tau)$.

Theorem 3.18. Suppose $(V, j) \models \mathrm{ZFR}$ and let $\tau$ be a limit ordinal and $\chi=$ $\operatorname{eot}(\tau)$. Let $J=\bigcap_{\xi<\tau} N_{\xi}$. Let $\xi<\tau$ be such that $\chi$ is $(\xi, \tau)$-stable and either

(i) $\chi<\operatorname{cr}\left(j_{\xi}\right)$, or

(ii) $\kappa_{\xi}<\chi=\tau=\kappa_{\tau}=\sup _{\gamma<\tau} \kappa_{\gamma}$.

Let $\mu=\operatorname{cof}^{M_{\xi}}(\tau)=\operatorname{cof}^{M_{\xi}}(\chi)$, so $i_{\xi \gamma}(\mu)=\operatorname{cof}^{M_{\gamma}}(\chi)$ for all $\gamma \in[\xi, \tau)$. Then:

1. $J$ is transitive, proper class with $N_{\tau} \subseteq J$ and $\left(J, M_{\tau}\right) \models \mathrm{ZF}$.

2. if $\mu>\omega$ then $N_{\tau}=J$.

3. if $\mu=\omega$ then $N_{\tau} \subsetneq J \models " \operatorname{cof}(\gamma)=\omega$ for all $N_{\tau}$-regular $\gamma \in\left[\kappa_{\tau}, \kappa_{\tau+\omega}\right)$ ".

Proof. Part 1: $J$ is clearly transitive with $N_{\tau} \subseteq J$. And $\left(J, M_{\tau}\right)$ is a class of $M_{\xi}=\left(N_{\xi}, j_{\xi}\right)$ for each $\xi<\tau$. So $J$ satisfies Powerset: if $X \in J$ and $\xi<\tau$ then because $J$ is a class of $M_{\xi}$, we have $\mathcal{P}(X) \cap J \in M_{\xi}$; therefore $\mathcal{P}(X) \cap J \in J$. Likewise, for each $\alpha \in \mathrm{OR}$, we have $V_{\alpha} \cap J \in J$ and $\left\langle V_{\beta} \cap J\right\rangle_{\beta<\alpha} \in J$. Separation (with respect to the predicate $M_{\tau}$ ) is similar to Powerset, and Collection follows from the preceding remarks.

Part 2: Suppose $\mu>\omega$. Let $x \in J$. By Lemma 3.13 and transitivity, it suffices to see that $x$ is $\tau$-es. Suppose not. Then for each $\gamma<\tau$ there is $\gamma^{\prime} \in(\gamma, \tau)$ such that $i_{\gamma \gamma^{\prime}}(x) \neq x$.

Work in $M_{\xi}$, where $\operatorname{cof}(\chi)=\mu>\omega$. We have $\xi+\chi=\tau$. Define a sequence $\left\langle\xi_{n}\right\rangle_{n<\omega}$ as follows: Set $\xi_{0}=0$. Given $\xi_{n}$, let $\xi_{n+1}$ be the least $\xi^{\prime} \in\left(\xi_{n}, \eta\right)$ such that $\widetilde{i}_{\xi_{n} \xi^{\prime}}^{M_{\xi}}(x) \neq x$. Then since $\operatorname{cof}(\chi)>\omega$, we have $\left(\sup _{n<\omega} \xi_{n}\right)=\xi_{\omega}<\chi$. So $x \in \widetilde{N}_{\xi_{\omega}}$, so by Lemma 3.13, $x$ is $\xi_{\omega}$-es, a contradiction.

Part 3: Suppose $\mu=\omega$. Work in $M_{\xi}$. Let $\left\langle\xi_{n}\right\rangle_{n<\omega}$ with $\sup _{n<\omega} \xi_{n}=\chi$ and $\xi_{0}=0$. Define $\left\langle\xi_{n}^{\prime}\right\rangle_{1 \leq n<\omega}$ by $\widetilde{i}_{\xi_{n}}^{M_{\xi}}\left(\xi_{n}\right)$ and $\xi_{0}^{\prime}=0$. Note $\left\langle\xi_{n}^{\prime}\right\rangle_{n>m} \in M_{\xi_{m}^{\prime}}$ (by commutativity of the maps), so in fact $\left\langle\xi_{n}^{\prime}\right\rangle_{n<\omega} \in J$. Note $\xi_{n} \leq \xi_{n}^{\prime}<\xi_{n+1}^{\prime}<\chi$ (since $\chi$ is $(\xi, \tau)$-stable) and $\sup _{n<\omega} \xi_{n}^{\prime}=\sup _{n<\omega} \xi_{n}=\chi$.

Renaming, we may assume $\left\langle\xi_{n}\right\rangle_{n<\omega} \in J$. Now let $\gamma \in\left[\kappa_{\tau}, \kappa_{\tau+\omega}\right)$ be regular in $N_{\tau}$. We need $J \models$ "cof $(\gamma)=\omega$ ". We may assume $\gamma \in \operatorname{rg}\left(i_{\xi \tau}\right)$; let $i_{\xi \tau}(\bar{\gamma})=\gamma$. So $M_{\xi} \models$ " $\bar{\gamma}$ is regular". Let $\bar{\gamma}_{n}=i_{\xi, \xi+\xi_{n}}(\bar{\gamma})$, so $i_{\xi+\xi_{n}, \tau}\left(\bar{\gamma}_{n}\right)=\gamma$. Let

$$
\bar{\zeta}_{n+1}=\sup i_{\xi+\xi_{n}, \xi+\xi_{n+1}} " \bar{\gamma}_{n} .
$$


Now $\bar{\zeta}_{n+1}<\bar{\gamma}_{n+1}$. For $j_{\xi+\xi_{n}}\left(\gamma^{\prime}\right)>\gamma^{\prime}$, for all $\gamma^{\prime} \in\left[\kappa_{\xi+\xi_{n}}, \kappa_{\xi+\xi_{n}+\omega}\right)$. But $j_{\xi+\xi_{n}}: M_{\xi+\xi_{n}} \rightarrow M_{\xi+\xi_{n}}$, so by the regularity of $j_{\xi+\xi_{n}}\left(\bar{\gamma}_{n}\right)$ in $M_{\xi+\xi_{n}}$, and since $j_{\xi+\xi_{n}}\left\lceil\bar{\gamma}_{n} \in M_{\xi+\xi_{n}}\right.$, we get

$$
\left(\sup j_{\xi+\xi_{n}} " \bar{\gamma}_{n}\right)<j_{\xi+\xi_{n}}\left(\bar{\gamma}_{n}\right),
$$

and by commutativity, therefore $\bar{\zeta}_{n+1}<\bar{\gamma}_{n+1}$. Let $\zeta_{n+1}=i_{\xi+\xi_{n+1}, \tau}\left(\bar{\zeta}_{n+1}\right)$. Then $\sup _{n<\omega} \zeta_{n+1}=\gamma$, and note $\left\langle\zeta_{n+1}\right\rangle_{n<\omega} \in J$, using $\left\langle\xi_{n}\right\rangle_{n<\omega} \in J$.

\subsection{The (infinite) iterates $N_{\tau}$ are not set grounds of $V$}

In contrast to the fact that every set is generic over $N_{\tau}$, we have:

Theorem 3.19. Suppose $(V, j) \models \mathrm{ZFR}$. Then:

1. For all $\tau \in \mathrm{OR}$ with $\tau \geq \omega, N_{\tau}$ is not a set ground of $V$.

2. For $\xi<\tau \in \mathrm{OR}$, we have:

(a) $i_{\xi \tau} \backslash V_{\alpha}^{N_{\xi}} \in N_{\tau}$ for all $\alpha<\kappa_{\xi+\omega}$.

(b) If $\xi+\omega \leq \tau$ then $i_{\xi \tau}\left\lceil\kappa_{\xi+\omega} \notin N_{\tau}\right.$.

Proof. Part 2a: If $\tau<\xi+\omega$ this is immediate because $N_{\tau}=N_{\xi}$. So suppose $\xi+\omega \leq \tau$. Let $n<\omega$ be such that $\alpha<\kappa_{\xi+n}$. Then $k=i_{\xi, \xi+n}\left\lceil V_{\alpha}^{N_{\xi}} \in N_{\xi+n}=\right.$ $N_{\xi}$, and note $i_{\xi \tau}\left\lceil V_{\alpha}^{N_{\xi}}=i_{\xi+n, \tau} \circ k=i_{\xi+n, \tau}(k) \in N_{\tau}\right.$.

Part 2b: Suppose first $\tau=\xi+\omega$. Then $i_{\xi, \xi+\omega}$ " $\kappa_{\xi+\omega}$ is cofinal in $\kappa_{\xi+\omega+\omega}$, and $\operatorname{cof}^{N_{\xi+\omega}}\left(\kappa_{\xi+\omega}\right)=\kappa_{\xi+\omega}=\operatorname{cr}\left(j_{\xi+\omega}\right)$. But $\operatorname{cof}^{N_{\xi+\omega}}\left(\kappa_{\xi+\omega+\omega}\right)=\omega$, because $\kappa_{\xi+\omega+\omega}=\widetilde{\kappa}_{\omega}^{M_{\xi+\omega}}$. Therefore $i_{\xi, \xi+\omega}\left\lceil\kappa_{\xi+\omega} \notin N_{\xi+\omega}\right.$.

Now suppose $\tau>\xi+\omega$. Since $i_{\xi+\omega, \tau}$ is computed by $M_{\xi+\omega}$, and by commutativity, if $i_{\xi \tau}\left\lceil\kappa_{\xi+\omega} \in N_{\tau} \subseteq N_{\xi+\omega}\right.$, then $i_{\xi, \xi+\omega}\left\lceil\kappa_{\xi+\omega} \in N_{\xi+\omega}\right.$, a contradiction.

Part 1: By part 2, $i_{0 \tau}\left\lceil\kappa_{\omega}\right.$ is a set of ordinals in $V \backslash N_{\tau}$, and $i_{0 \tau}: V \rightarrow N_{\tau}$ is elementary. So by Theorem 2.3, $N_{\tau}$ is not a set-ground of $V$.

\subsection{Non-definability of $j\lceil\mathrm{OR}$}

Remark 3.20. Recall Suzuki [17] showed that no elementary $j: V \rightarrow V$ is definable from parameters. Variants of this are proved in [7], [6], [14]. In particular, [7, Theorem 35] establishes the stronger fact that no elementary $k: \mathrm{HOD} \rightarrow \mathrm{HOD}$ is definable from parameters (hence if $j: V \rightarrow V$ is elementary then $j\lceil\mathrm{OR}$ is not definable from parameters). We sketch now an alternate proof of this fact, closer to the methods of this paper. Suppose $k:$ HOD $\rightarrow$ HOD is definable from parameter $x$. Using Vopenka (or maybe a slight adaptation thereof to ZF, if it is not standard; see Fact 3.24), there is a HOD-set-generic filter $G_{x}$ such that $\operatorname{HOD}\left[G_{x}\right]=\operatorname{HOD}_{x} \cdot{ }^{12}$ So $k\left\lceil\operatorname{HOD}\right.$ is amenable to $\operatorname{HOD}\left[G_{x}\right]$. But this is impossible by [7, Corollary 9]; or alternatively, using Lemma 2.2, we get that $k\lceil\mathrm{HOD}$ is amenable to HOD, contradicting Kunen.

In this section we strengthen the fact that $j\lceil\mathrm{OR}$ is not definable from parameters, if $j: V \rightarrow V$ is elementary, showing $j\lceil$ OR cannot be definable from parameters over the structure $\left(V, M_{\omega}\right)$, that is, allowing $M_{\omega}$ as a predicate.

\footnotetext{
${ }^{12}$ We cannot expect that $x \in \operatorname{HOD}\left[G_{x}\right]$ in general, since $\operatorname{HOD}\left[G_{x}\right] \models \mathrm{ZFC}$, and $x$ is arbitrary. However, the usual arguments still give $\operatorname{HOD}\left[G_{x}\right]=\operatorname{HOD}_{x}$.
} 
Also, it is shown in [7, Theorem 12] under ZFC 2 that if $j: V \rightarrow M$ is an elementary with $M$ wellfounded, then $V=\operatorname{Hull}^{V}(\operatorname{rg}(j))$. But the proof relies strongly on AC. It trivially fails with respect to $j$ when $(V, j) \models \mathrm{ZFR}$ (we have $\operatorname{rg}(j)=\operatorname{Hull}^{V}(\operatorname{rg}(j))$ by elementarity). It also fails with respect to $j=i_{0 \omega}$, as shown in the following theorem (but since $N_{\omega} \neq V$, this time it is not trivial). Note that $\operatorname{Hull}^{M}(X)$ was defined in $§ 1.1$.

Theorem 3.21. Assume $(V, j) \models \mathrm{ZFR}$. Then:

1. For no set $X$ is $V=\operatorname{Hull}^{\left(V, M_{\omega}\right)}\left(N_{\omega} \cup X\right)$.

2. $j\left\lceil\mathrm{OR}\right.$ and $i_{0 \omega}\left\lceil\mathrm{OR}\right.$ are not definable from parameters over $\left(V, M_{\omega}\right)$.

Proof. Part 1: Suppose $X \in V$ and $V=\operatorname{Hull}^{\left(V, M_{\omega}\right)}\left(N_{\omega} \cup X\right)$. Let $k_{n}=i_{n \omega} \uparrow$ $X^{<\omega}$, for $n<\omega$. So $\operatorname{rg}\left(k_{n}\right) \subseteq N_{\omega}$. Let $\vec{k}=\left\langle k_{n}\right\rangle_{n<\omega}$. Then $(X, \vec{k}) \in V$, so by Theorem 3.9, we have $(X, \vec{k}) \in N_{\omega}[G]$ for some set-generic extension $N_{\omega}[G]$ of $N_{\omega}$. We will show that $N_{\omega}[G]=V$, contradicting Theorem 3.19.

Let $Y \in V$. By our contradictory assumption, there is $\alpha \in \mathrm{OR}$ and $n<\omega$ such that $Y$ is encoded into $t=\operatorname{Th}_{\Sigma_{n}}^{\left(V, M_{\omega}\right)}\left(\left(N_{\omega} \cap V_{\alpha}\right) \cup X\right)$. So it suffices to see $t \in N_{\omega}[G]$. We have $\left(N_{\omega} \cap V_{\alpha}\right) \cup \stackrel{X}{X} \in N_{\omega}[G]$, so we just need to verify the appropriate instance of Separation.

So let $\varphi$ be $\Sigma_{n}$ and $\vec{y} \in\left(N_{\omega} \cap V_{\alpha}\right)^{<\omega}$ and $\vec{x} \in X^{<\omega}$. For each $m<\omega$, note

$$
\left[\left(V, M_{\omega}\right)=\left(N_{m}, M_{\omega}\right) \models \varphi(\vec{y}, \vec{x})\right] \Longleftrightarrow\left[\left(N_{\omega}, M_{\omega+\omega}\right) \models \varphi\left(i_{m \omega}(\vec{y}), i_{m \omega}(\vec{x})\right)\right] .
$$

But $i_{m \omega}(\vec{x})=k_{m}(\vec{x})$ for all $m<\omega$, and $i_{m \omega}(\vec{y})=\vec{y}^{*}=i_{\omega, \omega+\omega}(\vec{y})$ for all sufficiently large $m<\omega$. Therefore

$$
\left[\left(V, M_{\omega}\right) \models \varphi(\vec{y}, \vec{x})\right] \Longleftrightarrow \exists \ell<\omega \forall m \geq \ell\left[\left(N_{\omega}, M_{\omega+\omega}\right) \models \varphi\left(\vec{y}^{*}, k_{m}(\vec{x})\right)\right] .
$$

Since $\left\langle k_{m}\right\rangle_{m<\omega} \in N_{\omega}[G]$ and $i_{\omega, \omega+\omega} \uparrow\left(N_{\omega} \cap V_{\alpha}\right) \in N_{\omega}$, we get $t \in N_{\omega}[G] .{ }^{13}$

Part 2: Arguing as above, note that for each $x \in V$, letting $x_{n}=i_{n \omega}(x)$, and letting $N_{\omega}[G]$ be a set-generic extension such that $\left\langle x_{n}\right\rangle_{n<\omega} \in N_{\omega}[G]$, we get that every set $A \subseteq N_{\omega}$ which is definable over $\left(V, M_{\omega}\right)$ from parameters in $N_{\omega} \cup\{x\}$, is in $N_{\omega}[G]$.

Now suppose $i_{0 \omega}\left\lceil\mathrm{OR}\right.$ is definable over $\left(V, M_{\omega}\right)$ from elements of $N_{\omega} \cup\{x\}$. Then by the previous paragraph, $i_{0 \omega}\left\lceil\mathrm{OR}\right.$ is amenable to $M_{\omega}[G]$. But then much as in the proof of Lemma 2.2, we can show that $i_{0 \omega}\lceil\mathrm{OR}$ is amenable to $M_{\omega}$, contradicting Theorem 3.19. That is, fix $\alpha \in \mathrm{OR}$. We can find an $X \in M_{\omega}$ and $\beta \in \mathrm{OR}$ with $X \subseteq \beta$ and $X$ of ordertype $\alpha$, such that $i_{0 \omega}\left\lceil\alpha \in M_{\omega}\right.$. Let $\pi: \alpha \rightarrow X$ be the increasing enumeration. Then $i_{0 \omega}(\pi), i_{0 \omega}(X) \in N_{\omega+\omega} \subseteq N_{\omega}$, and $i_{0 \omega}(\pi): i_{0 \omega}(\alpha) \rightarrow i_{0 \omega}(X)$ is the increasing enumeration of $i_{0 \omega}(X)$. Since $i_{0 \omega} \circ \pi=i_{0 \omega}(\pi) \circ i_{0 \omega}\left\lceil\alpha\right.$, and $i_{0 \omega}\left\lceil X=\operatorname{rg}(\pi) \in N_{\omega}\right.$, we therefore get $i_{0 \omega}\left\lceil\alpha \in N_{\omega}\right.$, as desired.

Finally suppose $j\left\lceil\mathrm{OR}\right.$ is definable over $\left(V, M_{\omega}\right)$ from elements of $N_{\omega} \cup\{x\} .{ }^{14}$ Note $j\left\lceil\kappa_{\omega} \notin N_{\omega}\right.$, since otherwise $\operatorname{cof}^{N_{\omega}}\left(\kappa_{\omega}\right)=\omega$. But $j \uparrow N_{\omega}: M_{\omega} \rightarrow M_{\omega}$ is

\footnotetext{
${ }^{13}$ Of course, in $N_{\omega}[G]$ we can compute the relevant truth of $\left(N_{\omega}, M_{\omega+\omega}\right)$, just noting that whenever $\left(V_{\xi}, V_{\xi} \cap M_{\omega}\right) \preccurlyeq_{n}\left(V, M_{\omega}\right)$, then $\left(V_{i_{0 \omega}(\xi)}^{N_{\omega}}, V_{i_{0 \omega}(\xi)}^{N_{\omega}} \cap M_{\omega+\omega}\right) \preccurlyeq_{n}\left(N_{\omega}, M_{\omega+\omega}\right)$. (In the notes [16], at this point it was claimed that we could alternatively use the definability of set-grounds here to allow $N_{\omega}[G]$ to compute $N_{\omega}$, but this was an oversight, since AC fails, and it is not clear to the author whether one can get around that failure here.)

${ }^{14}$ Note this doesn't immediately contradict the previous paragraph, because from $j\lceil\mathrm{OR}$ alone it doesn't seem that one can directly compute $i_{0 \omega}\left\lceil\mathrm{OR}\right.$. We can compute $i_{0 n}\lceil\mathrm{OR}$ for $n<\omega$, since this is the same as $j \circ \ldots \circ j \uparrow \mathrm{OR}$. But for $i_{0 \omega} \uparrow \mathrm{OR}$ it seems we need the direct limit of all $j_{n}\lceil\mathrm{OR}$.
} 
elementary. So we can just use essentially the same argument as in the previous paragraph for a contradiction (but slightly easier).

Remark 3.22. An immediate corollary is that there is no $X \in V$ such that $V=\operatorname{HOD}(X)$. And if $\left(V_{\delta}, V_{\delta+1}\right) \models \mathrm{ZF}_{2}+" \delta$ is total Reinhardt", then there is no $A \subseteq V_{\delta}$ and $X \in V_{\delta}$ such that $V_{\delta}=\left(\operatorname{HOD}_{A}(X)\right)^{\left(V_{\delta}, A\right)}$. For just let $j:\left(V_{\delta},(A, X)\right) \rightarrow\left(V_{\delta},(A, X)\right)$ be elementary, and run the obvious variant of the preceding proof.

But suppose $(V, j) \models$ ZFR. In the notes [16, v1 on arxiv.org], the author asked whether possibly $V=\operatorname{Hull}^{V}(\mathrm{OR} \cup \operatorname{rg}(j))$, or $V=\operatorname{Hull}^{V}\left(M_{\omega} \cup \operatorname{rg}(j)\right)$, and whether AC can be set-forceable over $V$. These questions have since been answered: Goldberg [5] and Usuba [19] have independently shown that AC is not set-forceable (not by considering the iterates $M_{\alpha}$, but by a more direct combinatorial method). In particular, there is no class $A$ and set $X$ such that $V=\operatorname{HOD}_{A}(X)$. Goldberg's proof also gives that if there is a Berkeley cardinal then AC is not set-forceable, and he also showed that (i) $V=\operatorname{Hull}^{V}\left(M_{\omega} \cup \operatorname{rg}(j)\right)$, but (ii) $V \neq \operatorname{Hull}^{V}(\mathrm{OR} \cup \operatorname{rg}(j))$.

By an easier argument than that for (i), we have $\operatorname{Hull}^{(V, j)}\left(N_{\omega}\right)=V$, in contrast to the fact that $\operatorname{Hull}^{\left(V, M_{\omega}\right)}\left(N_{\omega}\right)=N_{\omega}$. For $i_{0 \omega}$ is computable from $j$, and given $x \in V$, we have $x=\left(i_{0 \omega}\right)^{-1}\left(x^{\prime}\right)$ where $x^{\prime}=i_{0 \omega}(x) \in N_{\omega}$.

Remark 3.23. Assume ZF. Let $x \subseteq V_{\alpha}$. We recall the definition of the Vopenka algebra Vop for extending $\operatorname{HOD}$ generically to $\operatorname{HOD}_{x}=\operatorname{HOD}(\{x\})$. Define Vop' in $V$ as follows. Conditions are non-empty OD subsets of $V_{\alpha+1}$; the ordering is $A \leq B \Longleftrightarrow A \subseteq B$. Then Vop $\in$ HOD is the natural coding of Vop' as a set of ordinals. Let $\pi:$ Vop $\rightarrow$ Vop' $^{\prime}$ be the natural isomorphism. Now (with $x \subseteq V_{\alpha}$ ), define the filter $G_{x}=\{p \in \operatorname{Vop} \mid x \in \pi(p)\}$.

Then by the ZFC arguments, we have:

Fact 3.24. $G_{x}$ is (HOD, Vop)-generic and $\operatorname{HOD}\left[G_{x}\right]=\mathrm{HOD}_{x} \cdot{ }^{15}$

Although every set is set-generic over $N_{\tau}$ for every $\tau<\mathrm{OR}$, this fails at $\tau=\mathrm{OR}$, and although $\mathrm{HOD} \subseteq N_{\omega}$, there is $\tau<\mathrm{OR}$ with $\mathrm{HOD} \nsubseteq N_{\tau}$ :

Theorem 3.25. Suppose $(V, j) \models \mathrm{ZFR}$. Let $J=\bigcap_{\alpha \in \mathrm{OR}} N_{\alpha}$. Then:

1. $J$ is a transitive proper class and $V_{\kappa_{\tau}}^{N_{\tau}} \preccurlyeq V_{\kappa_{\tau+\omega}}^{N_{\tau}} \preccurlyeq J$ for each $\tau \in \mathrm{OR}$.

2. For all $\alpha \leq \beta<\mathrm{OR}, i_{\alpha \beta}\lceil J: J \rightarrow J$ is elementary.

3. $j\left\lceil V_{\kappa_{\omega}}^{J}: V_{\kappa_{\omega}}^{J} \rightarrow V_{\kappa_{\omega}}^{J}\right.$ is not set-generic over $J$.

4. $\mathrm{HOD} \nsubseteq J$.

Proof. Part 1 is straightforward, noting in particular that $V_{\kappa_{\tau+\omega}}^{N_{\tau}}=V_{\kappa_{\tau+\omega}}^{N_{\tau+\omega}} \subseteq J$.

Part 2: Note that $J$ is defined in the same manner over $M_{\alpha}=\left(N_{\alpha}, j_{\alpha}\right)$ as over $M_{\beta}$, so $i_{\alpha \beta}(J)=J$.

15 The fact that $x \in \operatorname{HOD}\left[G_{x}\right]$, in the case that $x \subseteq \gamma \in \mathrm{OR}$, is of course due to Vopenka. The author is not sure who first proved the entire result. Let $\varphi$ be a formula, $\eta, \gamma \in \mathrm{OR}$ and $A=\{\xi<\gamma \mid \varphi(x, \xi, \eta)\}$. We need $A \in \operatorname{HOD}\left[G_{x}\right]$. For $\xi<\gamma$ let $t_{\xi}^{\prime}=\{y \mid y \in$ $V_{\alpha+1}$ and $\left.\varphi(y, \xi, \eta)\right\}$. So $t_{\xi}^{\prime} \in \operatorname{Vop}^{\prime}$. Let $\pi\left(t_{\xi}\right)=t_{\xi}^{\prime}$. Note $\left\langle t_{\xi}\right\rangle_{\xi<\gamma} \in$ HOD, and $t_{\xi} \in G_{x}$ iff $x \in t_{\xi}^{\prime}$ iff $\xi \in X$, so $X \in \operatorname{HOD}\left[G_{x}\right]$. 
Part 3: Let $R=\left(V_{\kappa_{\omega}}, j \uparrow V_{\kappa_{\omega}}\right)$. Note $R$ is iterable with iterates $R_{\alpha}=$ $\left(V_{\kappa_{\alpha+\omega}}^{M_{\alpha}}, j_{\alpha}\left\lceil V_{\kappa_{\alpha+\omega}}^{M_{\alpha}}\right)=\left(V_{\kappa_{\alpha+\omega}}^{J}, j_{\alpha}\left\lceil V_{\kappa_{\alpha+\omega}}^{J}\right)\right.\right.$. But then if $R \in J[G]$, we can form the iteration $\left\langle R_{\alpha}\right\rangle_{\alpha \in \mathrm{OR}}$ there, and considering some sufficiently large limit $\alpha$, this collapses all cardinals in $\left(\kappa_{\alpha}, \kappa_{\alpha+\omega}\right]$ in $J[G]$, although each $\kappa_{\alpha+n+1}$ is inaccessible in $J$ and hence also in $J[G]$, a contradiction.

Part 4: Let $G$ be $\left(V, \operatorname{Col}\left(\omega, V_{\kappa_{\omega}}\right)\right)$-generic. Let $H=\operatorname{HOD}^{V[G]}$. By the homogeneity of the collapse, $H \subseteq$ HOD. Let $x \in V[G]$ be a real coding $R$. Then by Fact $3.24, R \in H\left[G_{x}\right]=\operatorname{HOD}_{x}^{V[G]}$ is a set-generic extension of $H$. Let $\xi \in \mathrm{OR}$ with $\operatorname{Vop}^{V[G]} \subseteq \xi$. So $\left(\xi^{+n+1}\right)^{H\left[G_{x}\right]}=\left(\xi^{+n+1}\right)^{H}$. Since $R \in \mathrm{HC}^{H\left[G_{x}\right]}$, in $H\left[G_{x}\right]$ we can iterate $R$, and $R_{\xi^{+H}}$ has cardinality $\xi^{+H}$ there, and $\xi^{+H}=\kappa_{\xi^{+H}}$. So $\mathcal{P}\left(\xi^{+H}\right) \cap J$ also has cardinality $\xi^{+H}$ there. So $H \nsubseteq J$.

\subsection{No Berkeley cardinal in $\operatorname{HOD}_{A}(X)$}

We now adapt the proof of the previous section to show that if there is a Berkeley cardinal (see [1]) then there is no class $A$ and set $X$ such that $V=\operatorname{HOD}_{A}(X)$. However, as mentioned in Remark 3.22, one can prove much more.

Theorem 3.26. Let $\Omega \in \mathrm{OR}$ be such that $V_{\Omega} \models \mathrm{ZF}+$ "There is a Berkeley cardinal". Then there is no $A \subseteq V_{\Omega}$ such that $\left(V_{\Omega}, A\right) \models \mathrm{ZF}(A)+$ "V $=\operatorname{HOD}_{A}(X)$ for some set $X "$.

Proof sketch. Suppose otherwise and work in $\left(V_{\Omega}, A\right)$. Let $\beta$ be Berkeley. Let $\lambda \in \mathrm{OR}, \lambda>\beta$, be such that $\left(V_{\lambda}, A \cap V_{\lambda}\right) \preccurlyeq 10(V, A)$ and $\operatorname{cof}(\lambda)>\omega$ (note $\beta$ is a limit of inaccessibles, so there are certainly ordinals of cofinality $>\omega)$. Let

$$
j:\left(V_{\lambda}, A \cap V_{\lambda}\right) \rightarrow\left(V_{\lambda}, A \cap V_{\lambda}\right)
$$

be elementary with $\operatorname{cr}(j)<\beta$. By elementarity, there is $X \in V_{\lambda}$ such that $\left(V_{\lambda}, A \cap V_{\lambda}\right) \models " V=\operatorname{HOD}_{A}(X)$ ", where here $\operatorname{HOD}_{A}(X)$ is defined locally from $A$; that is, for every $x \in V_{\lambda}$, there is $\xi<V_{\lambda}$ such that $x$ is definable over $\left(V_{\xi}, A \cap V_{\xi}\right)$ from parameters in $X \cup \xi$.

Now we adapt the proof in the Reinhardt cardinal context to $\left(V_{\lambda}, A \cap V_{\lambda}, j\right)$. We have $j \uparrow V_{\alpha} \in V_{\lambda}$ for each $\alpha<\lambda$, and $j$ " $V_{\lambda}$ is $\in$-cofinal in $V_{\lambda}$. Consider the structure $M_{0}=\left(V_{\lambda}, A \cap V_{\lambda}, j_{0}\right)$ where $j=j_{0}$. Define $M_{1}=j_{0}^{+}\left(M_{0}\right)$ as usual, and note $M_{1}=\left(V_{\lambda}, A \cap V_{\lambda}, j_{1}\right)$, where $j_{1}=j_{0}^{+}\left(j_{0}\right)$. There are unboundedly many $\alpha<\lambda$ of cofinality $\omega$ which are fixed by $j$. Fix such an $\alpha$. Note that $k=j_{0} \uparrow V_{\alpha}$ is fully elementary as a map

$$
k:\left(V_{\alpha}, A \cap V_{\alpha}, j_{0}\left\lceil V_{\alpha}\right) \rightarrow\left(V_{\alpha}, A \cap V_{\alpha}, j_{0}\left(j_{0}\left\lceil V_{\alpha}\right)\right) .\right.\right.
$$

Therefore $j: M_{0} \rightarrow M_{1}$ is cofinal $\Sigma_{1}$-elementary.

Also since these bounded fragments of $j_{0}$ are fully elementary, these facts are lifted by $j_{0}$, and so iterating, $j_{1}: M_{1} \rightarrow M_{2}$ is also cofinal $\Sigma_{1}$-elementary, etc. (In particular, $j_{1}$ also coheres $A$.) This proceeds through all $n<\omega$. Let $i_{m n}: M_{m} \rightarrow M_{n}$ be the iteration map.

Given $\alpha<\lambda$, let $\alpha^{\prime}=\sup _{n<\omega} i_{0 n}(\alpha)$. Then $\alpha^{\prime}<\lambda$, so note that the sequence $\vec{j}_{\alpha}=\left\langle i_{0 n}\left(j\left\lceil V_{\alpha}\right)\right\rangle_{n<\omega} \in V_{\lambda}\right.$. The map $\alpha \mapsto \vec{j}_{\alpha}$ is $\Sigma_{1}$-definable over $M_{0}$. It is now easy to adapt the usual proof to see that $M_{\omega}$ and the maps $i_{n \omega}: M_{n} \rightarrow M_{\omega}$ are uniformly $\Sigma_{1}$-definable over $M_{n}$, and $M_{\omega}$ is wellfounded, with $\mathrm{OR}^{M_{\omega}}=\lambda$.

We can now run the rest of the Reinhardt proof for a contradiction (of course using $\operatorname{HOD}_{A}(X)$ in place of $\operatorname{HOD}(X)$, which works because each $j_{n}$ coheres A). 


\section{Reinhardts and small forcing}

In this section we consider a different question regarding Reinhardt cardinals:

Question 4.1. Suppose $G$ is $(V, \mathbb{P})$-generic for some $\mathbb{P}$ in $V$, and $(V[G], j) \models$ ZFR. Is there a $k$ such that $(V, k) \models$ ZFR? Does $j \uparrow V$ work? What if we assume that $\mathbb{P} \in V_{\operatorname{cr}(j)}$ ? Note that the iterates $N_{\alpha}$ don't seem to help here, since $V$ is not a proper set-generic extension of $N_{\alpha}$.

We don't know the answer to this question in general, but we establish some related facts here. The forcing arguments in this section relate to Laver [10].

Definition 4.2. For a set $X, X$-AC is the statement that for every function $f$ with domain $X$, there is a choice function for $f$.

Remark 4.3. The following is a straightforward adaptation of the standard ground definability arguments of Laver, Woodin and Hamkins. Gitman and Johnstone also proved a related fact in [2, Main Theorem 1]. They show in particular that $(*)$ if $V \models \mathrm{ZF}+\delta$-DC, where $\delta$ is a cardinal, $\operatorname{card}(\mathbb{P})<\delta$, and $G$ is $(V, \mathbb{P})$-generic, then $V$ is definable over $V[G]$ from the parameter $\mathcal{P}(\delta)^{V}$. Actually, they prove something more general, in that they only assume that $\mathbb{P}$ admits a gap at $\delta$, not actually that $\operatorname{card}(\mathbb{P})<\delta$. We will prove below, however, a different generalization of $(*)$. The argument is very similar to both of those mentioned above.

Theorem 4.4. Let $M \models \mathrm{ZF}$ and $\mathbb{P} \in M$. Let $G$ be $(M, \mathbb{P})$-generic, and $C$ be the set of conditions of $\mathbb{P}$. Suppose $C$ is wellorderable in $M[G]$ and $M[G] \models$ " $\gamma+$-DC" where $\gamma=\operatorname{card}^{M[G]}(C)$. Then $M$ is definable over $M[G]$ from the parameter $\mathcal{P}\left(C^{2}\right)^{M} \cdot 16$

This improves on $(*)$ above, in that we do not assume that $M \models \gamma^{+}$-DC. (But of course, $[2]$ only demands that $\mathbb{P}$ admits a gap at $\delta$, not that $\operatorname{card}(\mathbb{P})<\delta$.)

Proof. Work in $M[G]$. Let $\lambda>\eta$ be a cardinal such that there is no surjection $f: V_{\alpha} \rightarrow \lambda$ with $\alpha<\lambda$. The following claim completes the proof:

Claim 3. $V_{\lambda} \cap M$ is the unique transitive $N \subseteq V_{\lambda}$ such that (i) $\lambda=\mathrm{OR} \cap N$, (ii) $V_{\alpha} \cap N \in N$ for each $\alpha<\lambda$, (iii) $N \models$ Separation, Pairing, Union, Powerset, (iv) $C \in N$ and $\mathcal{P}\left(C^{4}\right)^{N}=\mathcal{P}\left(C^{4}\right)^{M}$, (v) There is a partial order $\mathbb{Q} \in \mathcal{P}\left(C^{2}\right)^{N}$ and an $(N, \mathbb{Q})$-generic $H$ such that $V_{\lambda}=N[H]$.

Proof. We show by induction on $\xi$ that $V_{\xi}^{N}=V_{\xi}^{M}$. This is trivial by induction if $\xi$ is a limit. So assume it holds at $\xi \in[\omega, \lambda)$. Given $A \subseteq N$ and $B \in N$, we say that $A$ is $B$-amenable to $N$ iff $A \cap \operatorname{rg}(\pi) \in N$ for every function $\pi \in N$ such that $\pi: B \rightarrow N$.

Let $A \subseteq V_{\xi}$. We must show that $A \in M$ iff $A \in N$. We do this by proving:

1. $A \in M$ iff $A$ is $C^{2}$-amenable to $M$,

2. $A \in N$ iff $A$ is $C^{2}$-amenable to $N$,

3. for all $\pi: C^{2} \rightarrow V_{\xi}^{M}=V_{\xi}^{N}$, we have $\pi \in M$ iff $\pi \in N$.

\footnotetext{
${ }^{16}$ The author does not know whether $\mathcal{P}(C)^{M}$ suffices instead of $\mathcal{P}\left(C^{2}\right)^{M}$.
} 
Putting these things together, we get $A \in M$ iff $A \in N$, as desired.

Note first that we have the Forcing Theorem with respect to $\Sigma_{0}$ formulas, and definability of the Forcing Relation for $\Sigma_{0}$ formulas, over $V_{\lambda}^{M}$ and $N$; this follows from Separation and that $\lambda$ is closed enough.

Parts 1,2: It suffices to consider part 2. For the non-trivial direction, suppose that $A \notin N$; we show $A$ is not $C^{2}$-amenable to $N$. We have $V_{\lambda}=N[H]$. So let $\dot{A} \in N$ be such that $\dot{A}_{H}=A$. Since $A \subseteq V_{\xi}^{N}$ and $A \notin N$, there is $p_{0} \in H$ such that $N \models p_{0} \models_{\mathbb{Q}} " \dot{A} \subseteq \check{V}_{\xi}$ but $\dot{A} \notin \check{V} "$.

Now in $V$, where $\mathbb{Q} \subseteq C$ is wellordered and $|C|^{+}$-AC holds, let $f: C \rightarrow V_{\xi}^{N}$ be such that for each $p \leq{ }^{\mathbb{Q}} p_{0}, f(p)$ is some $x$ such that

$$
N \models p \text { does not decide (w.r.t. } \mathbb{Q} \text { ) whether } \check{x} \in \dot{A} \text {. }
$$

Let $\dot{f} \in N$ be such that $\dot{f}_{H}=f$. Let $p_{1} \in H, p_{1} \leq p_{0}$, such that $N \models$ $p_{1} \|_{\mathbb{Q}} " \dot{f}: \check{C} \rightarrow \check{V}_{\xi}$, and for each $p \leq \leq^{\check{\mathbb{Q}}} \check{p_{0}}, \check{V}$ thinks that $p$ does not decide whether $\dot{f}(p) \in \dot{A}$ ".

In $N$, define $f_{*}: C^{2} \rightarrow N$, where for $(p, q) \in C^{2}$, if $q \leq \mathbb{Q} p \leq \mathbb{Q} p_{1}$ then $f_{*}(p, q)$ is the unique $x$ such that $q \Vdash \leftarrow \dot{f}(\check{p})=\check{x}$, if there is such an $x$ (and say $(p, q)$ is $\operatorname{good})$, and $f_{*}(p, q)=\emptyset$ otherwise.

Let $R=\operatorname{rg}\left(f_{*}\right) \in N$. We claim that $R \cap A \notin N$, which completes the proof of part 2. So let $S \in \mathcal{P}(R)^{N}$. For each $p \leq p_{1}$ there is $q \leq p$ such that $(p, q)$ is good, and so $x=f_{*}(p, q) \in R$; but then there are also $p^{\prime} \leq p$ and $p^{\prime \prime} \leq p$ such that $p^{\prime} \Vdash \check{x} \in \dot{A}$ and $p^{\prime \prime} \Vdash \check{x} \notin \dot{A}$. So by density, there will be some such $x$ such that $x \in A$ iff $x \notin S$. So $R \cap A \neq S$, as desired.

Part 3: Let $\pi^{N}: C^{2} \rightarrow V_{\xi}^{M}=V_{\xi}^{N}$, with $\pi^{N} \in N$. Let $\pi^{M}: C^{2} \rightarrow V_{\xi}^{M}$, with $\pi^{M} \in M$. We show that $\pi^{N} \in M$ and $\pi^{M} \in N$.

Work in $M[G]$, where $|C|=\left|C^{2}\right|=\gamma$ and $\gamma^{+}$-DC holds. We construct a continuous increasing sequence $\left\langle X_{\alpha}\right\rangle_{\alpha<\gamma^{+}}$of sets such that $X_{0}=\operatorname{rg}\left(\pi^{N}\right)$, $X_{0} \cup \operatorname{rg}\left(\pi^{M}\right) \subseteq X_{1}, X_{\omega \alpha+2 n+1} \in M, X_{\omega \alpha+2 n+2} \in N, X_{\alpha} \subseteq V_{\xi}^{M}=V_{\xi}^{N}$, $\left|X_{\alpha}\right| \leq \gamma$ and $X_{\alpha}$ is extensional for each limit $\alpha$.

Given $X=X_{\omega \alpha+2 n}$, first let $Y \subseteq V_{\xi}^{M}$ be some set with $X \subseteq Y$ and $|Y|=\gamma$ and such that for all $x, y \in X$, if $x \neq y$ then there is $z \in Y$ such that $z \in x$ iff $z \notin y$. Then let $X_{\omega \alpha+2 n+1} \in M$ be some $Y^{\prime}$ such that $Y \subseteq Y^{\prime} \subseteq V_{\xi}^{M}$ and $Y^{\prime}$ is the surjective image of $W$ in $M$; this exists because $V_{\lambda}=V_{\lambda}^{M}[G]$, so $M$ can find a small covering set. For $X_{\omega \alpha+2 n+2}$ it is likewise. Make the sequence continuous. By $\gamma^{+}$-DC, we can proceed through $\gamma^{+}$sets; note that $\gamma$-DC ensures here that at limit stages $\alpha$, we still have $\left|X_{\alpha}\right|=\gamma$.

Now since $\lambda$ is large, the sequence is in $V_{\lambda}$. So let $\dot{X}^{N} \in N$ and $\dot{X}^{M} \in V_{\lambda}^{M}$ be names for it. Then we can find $p \in G$ such that for cofinally many $\alpha<\gamma^{+}$, in $M, p$ decides the value of $\dot{X}_{\alpha}$, and forces that the sequence is continuous. Let $D^{M} \in M$ be the cofinal set on which $p$ decides this. Then $\left\langle X_{\alpha}\right\rangle_{\alpha \in D} \in M$. Note that $D^{M}$ is closed. We get a similar club $D^{N} \in N$. Let $\alpha \in D^{M} \cap D^{N}$ be a limit ordinal. Then $X_{\alpha} \in M \cap N, X_{\alpha}$ is extensional, and $M, N$ both have surjections $C^{2} \rightarrow X_{\alpha}$ (since $M[G]$ has a surjection $C \rightarrow X_{\alpha+1}, M$ has one $C^{2} \rightarrow X_{\alpha+1}$, and likewise $N$ has one $\left.C^{2} \rightarrow X_{\alpha+2}\right)$.

Since $X=X_{\alpha}$ is extensional, we can let $\bar{X}$ be the transitive collapse of $X$, and $\pi: \bar{X} \rightarrow X$ the uncollapse map. Then $\bar{X}, \pi \in M \cap N$ (note the recursion defining the collapse maps take less than $\lambda$ many steps, by choice of $\lambda$ ). Note that $N, M$ have surjections from $C^{2}$ to $\bar{X}$. Since $\bar{X}$ is transitive, it follows 
that $\bar{X}$ is coded by subsets of $C^{4}$ in $M, N$. But $\mathcal{P}\left(C^{4}\right)^{M}=\mathcal{P}\left(C^{4}\right)^{N}$. So let $\sigma: C^{2} \rightarrow \bar{X}$ be a common surjection. Then $\pi \circ \sigma: C^{2} \rightarrow X$ is a common surjection. Now $\pi^{M} \in M$, so $(\pi \circ \sigma)^{-1} \circ \pi^{M} \in \mathcal{P}\left(C^{4}\right)^{M}=\mathcal{P}\left(C^{4}\right)^{N}$, so $\pi^{M} \in N$. Similarly $\pi^{N} \in M$.

This completes the proof of the theorem.

Theorem 4.5. Let $\eta \in \mathrm{OR}$ and $\mathbb{P} \subseteq V_{\eta}$ and $G$ be $(V, \mathbb{P})$-generic. Suppose $(V[G], j) \models$ ZFR where $\eta<\kappa=\operatorname{cr}(j)$, and $V[G] \models$ "X-AC" where $X=V_{\eta}$. Suppose $j\lceil V: V \rightarrow V$. Then $j$ is amenable to $V$.

Proof. Suppose not and let $\alpha \in \mathrm{OR}$ be such that $j\left\lceil V_{\alpha} \notin V\right.$. Let $\beta=j(\alpha)$. Let $\widetilde{k}$ be such that $\widetilde{k}_{G}=j\left\lceil V_{\alpha+5}\right.$. We may assume that $\mathbb{P}$ forces $" \widetilde{k}: V_{\alpha+5} \rightarrow V_{\beta+5}$ is elementary and $\widetilde{k}\left\lceil\check{V}_{\alpha} \notin \check{V}\right.$ ".

In $V[G]$, fix a function $f: \mathbb{P} \rightarrow V_{\alpha}^{V}$ such that $f(p)$ is some $x$ such that $V \models \neg \exists y\left[p \models_{\mathbb{P}} \widetilde{k}(\check{x})=\check{y}\right]$; such $f$ exists by $X$-AC in $V[G]$. Let $\widetilde{f} \in V$ be such that $\widetilde{f}_{G}=f$ and $V \models \mathbb{P} \Vdash \widetilde{f}: \check{\mathbb{P}} \rightarrow \check{V}_{\alpha}$.

In $V$, define the partial function $f_{*}: \mathrm{p} \mathbb{P} \times \mathbb{P} \rightarrow V_{\alpha}$ by

$$
f_{*}(p, q)=\text { unique } x \text { such that } q \models_{\mathbb{P}} \widetilde{f}(\check{p})=\check{x} \text {, }
$$

if $q \leq p$ and there is such an $x \in V_{\alpha}$, and $f^{*}(p, q)$ is undefined otherwise. Let $D=\operatorname{dom}\left(f_{*}\right)$.

Let $g_{*}=j\left(f_{*}\right) \in V$ and let $p_{0} \in \mathbb{P}$ be such that $V \models p_{0} \|_{\mathbb{P}} \widetilde{k}\left(\check{f}_{*}\right)=\check{g}_{*}$. Since $\mathbb{P} \in V_{\kappa}$ and by elementarity, $g_{*}: D \rightarrow V_{j(\alpha)}$ and $j \circ f_{*}=g_{*}$. Let $p_{1} \leq p_{0}$ with $V \models p_{1} \|_{\mathbb{P}} \widetilde{k} \circ \check{f}_{*}=\check{g_{*}}$. Fix $q \leq p_{1}$ with $\left(p_{1}, q\right) \in D$. Let $x=f_{*}\left(p_{1}, q\right)$. So by choice of $f, x, V \models \neg \exists y\left[p_{1} \models_{\mathbb{P}} \widetilde{k}(\check{x})=\check{y}\right]$. But then letting $y=g_{*}\left(p_{1}, q\right)$ we have $V \models p_{1} \models_{\mathbb{P}} \widetilde{k}(\check{x})=\check{y}$, contradiction.

We can now deduce that one cannot force a "new" Reinhardt cardinal $\kappa$ such that $\kappa$-DC holds in the generic extension with small forcing:

Theorem 4.6. Let $\mathbb{P} \in V$ and $G$ be $(V, \mathbb{P})$-generic and $C$ the set of $\mathbb{P}$-conditions. Suppose $(V[G], j) \models \mathrm{ZFR}+{ }^{\text {" } C}$ is wellordered and $\gamma^{+}$-DC holds where $\operatorname{card}(C)=$ $\gamma^{\prime \prime}$. Then $(V, j\lceil V) \models \mathrm{ZFR}$.

Proof. By 4.4, $V$ is definable over $V[G]$ from $V_{\eta+1}^{V}$, so $j\lceil V: V \rightarrow V$. So by $4.5, j$ is amenable to $V$. And $(V, j\lceil V)$ satisfies the ZF axioms because $(V[G], j)$ does and $(V, j\lceil V)$ is definable from parameters over $V[G]$.

Remark 4.7. Note that the converse direction is routine: If $(V, j) \models$ ZFR and $\mathbb{P} \in V_{\kappa}$ where $\kappa=\operatorname{cr}(j)$ and $G$ is $(V, \mathbb{P})$-generic, then $\left(V[G], j^{+}\right) \models$ ZFR where $j^{+}: V[G] \rightarrow V[G]$ extends $j$ with $j(G)=G$. It follows under $(V, P) \models$ $\mathrm{ZF}_{2}+$ "there is a super Reinhardt cardinal" then (since then there is a proper class of such) $(V[G], P[\mid G])$ satisfies the same, and an easy variant gives that if $(V, P) \models$ "OR is total Reinhardt", so does $(V[G], P[G])$. The other direction is less trivial.

Theorem 4.8. Assume $(V, P) \models \mathrm{ZF}_{2}$. Let $G$ be $(V, \mathbb{P})$-generic where $\mathbb{P} \in V$. Then: 
1. If $\kappa$ is super Reinhardt in $(V[G], P[G])$ then there is $\kappa^{\prime} \in \mathrm{OR}^{V}$ which is super Reinhardt in $(V, P)$.

2. If $\mathbb{P} \in V_{\delta}$ and $\left(V_{\delta}^{V[G]}, V_{\delta+1}^{V[G]}\right)$ is total Reinhardt, then $\left(V_{\delta}, V_{\delta+1}\right)$ is total Reinhardt.

Proof. Part 2: Let $\delta \in \mathrm{OR}$ with $\mathbb{P} \in V_{\delta}$ and $G$ be $(V, \mathbb{P})$-generic and suppose that $\left(V_{\delta}^{V[G]}, V_{\delta+1}^{V[G]}\right)$ is total Reinhardt. In $V$, fix $A \subseteq V_{\delta}$. In $V[G]$, let $\kappa<\delta$ be $\left(<\delta,\left(V_{\delta}, A\right)\right)$-reflecting with $\mathbb{P} \in V_{\kappa}$. Let

$$
j:\left(V_{\delta}[G],\left(V_{\delta}, A\right)\right) \rightarrow\left(V_{\delta}[G],\left(V_{\delta}, A\right)\right)
$$

be elementary with $\operatorname{cr}(j)=\kappa$. We claim that $j\left\lceil V_{\delta} \in V\right.$ and hence, $j$ witnesses what we need in $V$. It suffices to see that for each $\alpha<\delta$, we have $j\left\lceil V_{\alpha} \in V\right.$, because we have a name $j \in V$ for $j$, and $V_{\delta}$ is inaccessible, which gives $j \in V$ as usual.

So fix $\alpha<\delta$ with $\alpha=j(\alpha)$ and let $\dot{k} \in V_{\delta}$ be such that $\dot{k}_{G}=j\left\lceil V_{\alpha}\right.$. We may assume $\alpha>\kappa$. We may assume that $\mathbb{P}$ forces " $k: V_{\alpha} \rightarrow V_{\alpha}$ is elementary". Let $\xi \in(\alpha+\omega, \delta)$ with $\dot{k} \in V_{\xi}$. Note that the forcing facts in the following claim are defined from the relevant parameters over $V_{\xi}$ :

Claim 1. There are $Y, \sigma, \eta \in V$, with $Y \subseteq V_{\alpha}$ and such that (i) for each $p \in \mathbb{P}$, if there is $x \in V_{\alpha}$ such that $p$ does not decide (with respect to $\mathbb{P}$ ) the value of $\dot{k}(\check{x})$ then there is $x \in Y$ with this property, (ii) $\eta<\kappa$, and (iii) $\sigma: V_{\eta} \rightarrow Y$ is a surjection.

Proof. (The first part here is motivated by Usuba's discussion of LöwenheimSkolem cardinals in [18].) In $V[G]$, there is some $X \preccurlyeq V_{\xi}^{17}$ with $\mathbb{P}, \dot{k} \in X$ and such that the transitive collapse $\bar{X}$ of $X$ is in $V_{\kappa}^{V[G]}$. For in $V[G]$, let $\ell: V_{\delta}^{V[G]} \rightarrow V_{\delta}^{V[G]}$ be elementary with $\operatorname{cr}(\ell)=\kappa$ and $\ell(\kappa)>\xi$ and $\ell\left(V_{\delta}\right)=V_{\delta}$. Then $\ell^{\text {" }} V_{\xi} \preccurlyeq \ell\left(V_{\xi}\right)=V_{\ell(\xi)}$, and $\ell^{\text {" }} V_{\xi} \in V_{\delta}[G]$, and $\ell^{\text {" }} V_{\xi}$ has transitive collapse $V_{\xi} \in V_{j(\kappa)}^{V[G]}$. So $V_{\delta}[G] \models$ "There is $X \preccurlyeq V_{\ell(\xi)}$ with $\ell(\mathbb{P}, \dot{k}) \in X$ and such that

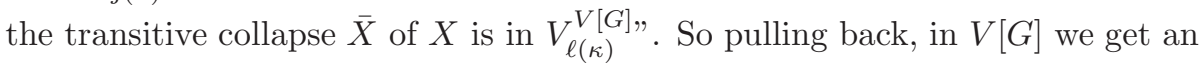
$X \preccurlyeq V_{\xi}$ with $\mathbb{P}, \dot{k} \in X$ and such that the transitive collapse $\bar{X}$ of $X$ is in $V_{\kappa}^{V[G]}$. Let $\dot{\bar{X}} \in V$ be a name for $\bar{X}$; we may take $\dot{\bar{X}} \in V_{\kappa}$. Let $\pi: \bar{X} \rightarrow X$ be the uncollapse map. Let $\dot{\pi} \in V$ be a name for $\pi$.

Now we may assume that $\mathbb{P}$ forces " $\dot{\pi}: \dot{\bar{X}} \rightarrow \check{V}_{\xi}$ ". Using the forcing relation in $V$, looking at all outputs forceable for $\dot{\pi}$, we can find some $Y \subseteq V_{\alpha}$ such that $X \cap V_{\alpha} \subseteq Y$ and $\eta<\kappa$ and a surjection $\sigma: V_{\eta} \rightarrow Y$ which works.

Let $\tau=j(\sigma) \in V$, so $\tau=j \circ \sigma$. Let $p \in G$ force " $\dot{j}: \check{V}_{\delta} \rightarrow \check{V}_{\delta}$ is elementary with $\operatorname{cr}(\dot{j})=\check{\kappa}$ and $\grave{j}\left\lceil\breve{V}_{\alpha}=\dot{k}\right.$ and $\check{\tau}=\dot{j}(\check{\sigma})=\dot{j} \circ \check{\sigma} "$. Note that $p$ forces the value of $\dot{k}(\check{x})=\dot{j}(\check{x})$ for every $x \in \operatorname{rg}(\sigma)$. But then by the claim, $p$ forces the value of $\dot{k}(\check{x})$ for every $x \in V_{\alpha}$. Therefore $j\left\lceil V_{\alpha} \in V\right.$, as desired.

Part 1: Let $V[G]$ be a set-generic extension such that $\kappa$ is super-Reinhardt in $V[G]$. Note then that if $j: V[G] \rightarrow V[G]$ is elementary with $\operatorname{cr}(j)=\kappa$, then $j(\kappa)$ is also super-Reinhardt in $V[G]$ (consider $j(k)$ for various $k$ ). And super Reinhardt easily implies supercompact. So (see [18]) $V[G] \models$ "There is

\footnotetext{
${ }^{17}$ We write $V_{\xi}$ for rank segments of $V$ and $V_{\xi}^{V[G]}$ for those of $V[G]$.
} 
a proper class of supercompact cardinals", so $V[G] \models$ "There is a proper class of Löwenheim-Skolem cardinals", so all set-grounds of $V[G]$ are definable from parameters over $V[G]$. Taking $\kappa^{\prime}$ super-Reinhardt then above the defining parameter, and $j^{\prime}: V[G] \rightarrow V[G]$ with $\operatorname{cr}\left(j^{\prime}\right)=\kappa^{\prime}$, it follows that $j^{\prime} \uparrow V: V \rightarrow V$. But then essentially the same argument as above shows that $j^{\prime}$ is amenable to $V$, as required.

Remark 4.9. Goldberg has shown [4, Theorem 6.12] that assuming ZF+DC $V_{\lambda+1}$ and $j: V_{\lambda+3} \rightarrow V_{\lambda+3}$ is $\Sigma_{1}$-elementary, one can prove Con(ZFC $\left.+I_{0}\right)$. It is not known at present, however, whether ZFR proves Con $\left(\mathrm{ZFC}+I_{0}\right)$.

Assume ZF $+k: V \rightarrow M$ is elementary with $M$ transitive, $\kappa=\operatorname{cr}(k)$, $\kappa_{1}=k(\kappa)$, and $k\left\lceil V_{\kappa_{1}} \in M\right.$, and

$$
j: V_{\lambda+3} \rightarrow V_{\lambda+3} \text { is } \Sigma_{1} \text {-elementary }
$$

with $\kappa<\operatorname{cr}(j)<\kappa_{\omega}(j) \leq \lambda<\kappa_{1}$. Then there is $\mathbb{P} \subseteq V_{\kappa}$ forcing $\mathrm{DC}_{V_{\lambda+1}}$, and $j$ extends $\Sigma_{1}$-elementarily to $j^{+}: V_{\lambda+3}^{V[G]} \rightarrow V_{\lambda+3}^{V[G]}$, so we can apply Goldberg's result above. To force $\mathrm{DC}_{V_{\lambda+1}}$, one can use a kind of argument used by Woodin to force DC from supercompacts. That is, let $\mathbb{P}$ be the finite support product of $\operatorname{Col}\left(\omega, V_{\alpha}\right)$, over all $\alpha<\kappa$. Let $G$ be $(V, \mathbb{P})$-generic. We claim $V[G] \models \mathrm{DC}_{V_{\alpha}}$ for all $\alpha<\kappa_{1}$, which suffices.

For first note that for every $x \in V_{\kappa_{1}}$, there is $\kappa^{\prime}<\kappa_{1}$ and a $\Sigma_{1}$-elementary $\pi: V_{\kappa} \rightarrow V_{\kappa^{\prime}}$ with $x \in \operatorname{rg}(\pi)$ and $\pi(\operatorname{cr}(\pi))=\kappa$. For $\pi^{\prime}=k \uparrow V_{\kappa_{1}}: V_{\kappa_{1}}^{M} \rightarrow V_{\kappa^{\prime}}^{M}$ is $\Sigma_{1}$-elementary where $\kappa^{\prime}=\sup k^{\prime \prime} \kappa_{1}$, and $k(x) \in \operatorname{rg}\left(\pi^{\prime}\right)$, and $\pi^{\prime}\left(\operatorname{cr}\left(\pi^{\prime}\right)\right)=\kappa_{1}$, and $\pi^{\prime} \in M$. So the existence of such a map $\pi^{\prime}$ pulls back under $k$, yielding a $\pi$ as desired.

Now let $R \in V_{\kappa_{1}}^{V[G]}$ be a finitely extendible relation, and $\dot{R} \in V_{\kappa_{1}}$ with $\dot{R}_{G}=R$. Let $\pi: V_{\kappa} \rightarrow V_{\kappa^{\prime}}$ be $\Sigma_{1}$-elementary with $\dot{R}_{G} \in \operatorname{rg}(\pi)$ and $\pi(\bar{\kappa})=\kappa$ where $\bar{\kappa}=\operatorname{cr}(\pi)$. Let $\pi(\overline{\mathbb{P}}, \overline{\dot{R}})=(\mathbb{P}, \dot{R})$ and $\bar{G}=G \cap V_{\bar{\kappa}}$. Note that every dense subset of $\overline{\mathbb{P}}$ in $V_{\kappa}$ is a pre-dense subset of $\mathbb{P}$, so $\bar{G}$ is $\left(V_{\kappa}, \overline{\mathbb{P}}\right)$-generic. Note that $\pi$ extends to a $\Sigma_{1}$-elementary $\pi^{+}: V_{\kappa}[\bar{G}] \rightarrow V_{\kappa^{\prime}}[G]$ with $\pi^{+}(\bar{G})=G$. Let $\bar{R}=\overline{\dot{R}}_{\bar{G}}$. So $\pi^{+}(\bar{R})=R$. But $\bar{R}$ is countable in $V[G]$, so $V[G]$ has an infinite branch $b$ through $\bar{R}$, and $\pi$ " $b$ is a branch through $R$.

Recall that by $[1], \mathrm{ZF}+$ " $\delta_{0}$ is the least Berkeley cardinal" implies the failure of $\operatorname{cof}\left(\delta_{0}\right)$-DC fails (in fact of $\operatorname{cof}\left(\delta_{0}\right)$-AC). If $j: V \rightarrow V$ is elementary then as mentioned in $[6]$ and $\S 3.2, \kappa_{\omega}(j)$ is somewhat Berkeley. These and the other considerations in this section lead to the following conjecture:

Conjecture 4.10. ZFR + DC has consistency strength strictly greater than that of ZFR.

\section{Competing Interests}

The author has no competing interests.

\section{Acknowledgements}

Funded by the Deutsche Forschungsgemeinschaft (DFG, German Research Foundation) under Germany's Excellence Strategy EXC 2044-390685587, Mathematics Münster: Dynamics-Geometry-Structure. 
The author thanks Gabriel Goldberg and Toshimichi Usuba for their answers to certain questions and helpful feedback.

\section{References}

[1] Joan Bagaria, Peter Koellner, and W. Hugh Woodin. Large cardinals beyond choice. Bulletin of Symbolic Logic, 25, 2019.

[2] Victoria Gitman and Thomas A. Johnstone. On ground model definability. In Infinity, Computability, and Metamathematics: Festschrift in honour of the 60th birthdays of Peter Koepke and Philip Welch, Series: Tributes. College publications, London, GB, 2014. arXiv:1311.6789.

[3] Victoria Gitman and Ralf Schindler. Virtual large cardinals. Annals of Pure and Applied Logic, 169(12), 2018.

[4] Gabriel Goldberg. Even ordinals and the Kunen inconsistency. arXiv:2006.01084.

[5] Gabriel Goldberg. Personal communication, 2020.

[6] Gabriel Goldberg and Farmer Schlutzenberg. Periodicity in the cumulative hierarchy. arXiv: 2006.01103.

[7] Joel David Hamkins, Greg Kirmayer, and Norman Lewis Perlmutter. Generalizations of the kunen inconsistency. Annals of Pure and Applied Logic, 163(12), 2012.

[8] Akihiro Kanamori. The higher infinite: large cardinals in set theory from their beginnings. Springer monographs in mathematics. Springer-Verlag, second edition, 2005.

[9] Kenneth Kunen. Elementary embeddings and infinitary combinatorics. Journal of Symbolic Logic, 36(3), 1971.

[10] Richard Laver. Certain very large cardinals are not created in small forcing extensions. Annals of Pure and Applied Logic, 2007.

[11] W. N. Reinhardt. Topics in the metamathematics of set theory. PhD thesis, UC Berkeley, 1967.

[12] W. N. Reinhardt. Remarks on reflection principles, large cardinals, and elementary embeddings. In Axiomatic set theory (Proc. Sympos. Pure Math., Vol. XIII, Part II, Univ. California, Los Angeles, Calif., 1967), pages 189205. Amer. Math. Soc., Providence, R. I., 1974.

[13] Grigor Sargsyan, Ralf Schindler, and Farmer Schlutzenberg. Varsovian models II. In preparation.

[14] Farmer Schlutzenberg. Extenders under ZF and constructibility of rankinto-rank embeddings. arXiv: 2006.10574.

[15] Farmer Schlutzenberg. On the consistency of ZF with an elementary embedding from $V_{\lambda+2}$ into $V_{\lambda+2}$. arXiv: 2006.01077. 
[16] Farmer Schlutzenberg. Reinhardt cardinals and non-definability (draft 1). arXiv: 2002.01215v1, notes.

[17] Akira Suzuki. No elementary embedding from $V$ into $V$ is definable from parameters. Journal of Symbolic Logic, 64(4), 1999.

[18] Toshimichi Usuba. Choiceless Löwenheim-Skolem property and uniform definability of grounds. arXiv: 1904.00895, 2019.

[19] Toshimichi Usuba. Personal communication, 2020.

[20] W. Hugh Woodin. Suitable extender models II: Beyond $\omega$-huge. Journal of Mathematical Logic, 11(2), 2011. 